\title{
ON ASYMPTOTIC STABILIZABILITY OF DISCRETE-TIME LINEAR SYSTEMS WITH DELAYED INPUT*
}

\author{
ZONGLI $\operatorname{LIN}^{\dagger}$
}

\begin{abstract}
This paper examines the asymptotic stabilizability of discrete-time linear systems with delayed input. By explicit construction of stabilizing feedback laws, it is shown that a stabilizable and detectable linear system with an arbitrarily large delay in the input can be asymptotically stabilized by either linear state or output feedback as long as the open loop system is not exponentially unstable (i.e., all the open loop poles are on or inside the unit circle.) It is further shown that such a system, when subject to actuator saturation, is semi-globally asymptotically stabilizable by linear state or output feedback.
\end{abstract}

Key words: Time delay, linear systems, stabilization, low gain feedback.

1. Introduction. This paper examines the asymptotic stabilizability of a discrete-time linear system with delay in the input,

$$
\left\{\begin{aligned}
x(k+1) & =A x(k)+B u(k-r), \quad x(s)=\phi(s), s \in[-r, 0], \\
y(k) & =C x(k),
\end{aligned}\right.
$$

where $x \in \mathbf{R}^{n}$ is the state, $u \in \mathbf{R}^{m}$ is the control input of either state feedback or output feedback type, $y \in \mathbf{R}^{p}$ is the measurement output, and $r \geq 0$ an integer that represents time delay in the control input. It is also assumed that the pair $(A, B)$ is stabilizable and the pair $(A, C)$ is detectable.

Control problems for linear time delay systems in the form of (1) or in a variety of other forms have been a subject of extensive research (see, for example, [1, 2, 3, $4,5,6,9,10,13,15,16,18,19,20,22,23,24,25,26]$ and the references there in). Various stability and stabilizability conditions were identified and stabilizing feedback laws constructed.

Our interest in discrete-time systems of the form (1) has been motivated by several recent results on asymptotic stabilization of their continuous-time counterparts,

$$
\left\{\begin{array}{l}
\dot{x}(t)=A x(t)+B u(t-\tau), \quad x(\theta)=\phi(\theta), \theta \in[-\tau, 0], \\
y(t)=C x(t),
\end{array}\right.
$$

where $\tau \geq 0$ is the delay. In particular, two special classes of (2) were recently revisited in $[13,8]$ and $[14]$, respectively. Both [13] and [8] showed that, in the presence

\footnotetext{
${ }^{*}$ This work was supported in part by National Natural Science Foundation of China under the Outstanding Young Investigator Program (Class B: Overseas Collaboration).

${ }^{\dagger}$ Charles L. Brown Department of Electrical and Computer Engineering, University of Virginia, P.O. Box 400743, Charlottesville, VA 22904-4743. E-mail: zl5y@virginia.edu
} 
of input delay, an oscillator system, which has a pair of open loop poles at $\pm j$, is globally asymptotically stabilizable by bounded state feedback laws. In [14], the authors established global asymptotic stablizability by bounded state feedback of a chain of integrators with a delay in the input.

While both the oscillator and the chain of integrator systems are special classes of (2) and both have all poles on the imaginary axis, the feedback laws that were constructed for stabilizing them are very different and the proof of the closed-loop stability involves different techniques. The feedback laws in [13] and [8] both involve a saturation function and both require the explicit knowledge of the amount of the delay. The closed-loop stability in [13] was established through that of a system under distributed control, while [8] resorted to analysis of trajectories. The feedback law in [14] does not require explicit knowledge of the amount of delay but involves a set of nested saturation functions and is thus nonlinear. Recently, the results of [14] were extended to open loop systems with all poles located on the closed left-half plane [25], where $L_{p}$ stabilization is also considered.

More recently, a general result on stabilizability of the system (2) was established in [12]. By explicit construction of stabilizing feedback laws, it was shown that system (2), with an arbitrarily large finite delay, is asymptotically stabilizable by either linear state or output feedback as long as the open loop system is not exponentially unstable (i.e., all the open loop poles are in the closed left-half plane). It is further shown that such systems, when subject to actuator saturation, are semi-globally asymptotically stabilizable by either linear state or output feedback.

This recent literature in a way indicates the complexity in the stabilization of systems with delay in the input such as those in the form of (2) and (1). The objective of this paper is to establish parallel results of [12] in the discrete-time setting. That is, the system (1), with an arbitrarily large finite delay, is asymptotically stabilized by either linear state or output feedback as long as the open loop system is not exponentially unstable (i.e., all the open loop poles are inside or on the unit circle), and such a system, when subject to actuator saturation, is semi-globally asymptotically stabilizable by either linear state or output feedback. Key to establishing these discrete-time results are the low gain feedback design technology [17] and the discrete-time version of the Razumikhin Theorem for delay difference equations [7, 27]. Even though these results are parallel to their continuous-time counterparts [12], their developments are more involved. For example, in the countinuous-time setting, the subsystems corresponding open loop poles at $s=0$, on the slow time scale, are independent of the low gain parameter $\varepsilon$. On the other hand, in the discrete-time setting, the subsystems corresponding to the open loop poles at $z=1$ or $z=-1$, on the slow time scale, contain terms that are in the order of $\varepsilon$. These $\varepsilon$ order perturbation terms lead to 
delicate analysis as the convergence rate of the closed-loop system under low gain feedback is also only the order of $\varepsilon$.

Simple examples show that the above mentioned results would not be true if the open loop system is exponentially unstable and thus are not conservative. For the continuous-time counterparts [12], we give some intuitive arguments as to why the knowledge of the delay is needed in the feedback law when there are non-zero open loop system poles at the imaginary axis. Here in the discrete-time setting, we will be able to come up with simple systems with open loop poles located on the unit circle but at $z \neq 1$ for which no delay independent linear feedback can achieve asymptotic stabilization.

The remainder of this paper is organized as follows. Section 2 contains some preliminaries that we need to establish our main results. Sections 3 contains state feedback results. Section 4 contains output feedback results. Section 5 deals with the situation when the inputs are subject to both delay and saturation. Section 6 includes some examples to demonstrate the results of the paper. Section 7 draws a conclusion to the paper.

Throughout the paper, we use rather standard notation. $\mathbf{R}$ denotes the set of real numbers, $\mathbf{R}^{n}$ denotes the set of $n$-dimensional real vectors, $\mathbf{R}^{n \times m}$ denotes the set of $n \times m$ real matrices, and $I_{n}$ denotes an $n \times n$ identity matrix.

\section{Preliminaries.}

2.1. Low Gain Feedback. Consider the following linear system,

$$
x(k+1)=A x(k)+B u(k), \quad x \in \mathbf{R}^{n}, u \in \mathbf{R},
$$

where

$$
A=\left[\begin{array}{ccccc}
0 & 1 & 0 & \cdots & 0 \\
0 & 0 & 1 & \cdots & 0 \\
\vdots & \vdots & \vdots & \ddots & \vdots \\
0 & 0 & 0 & \cdots & 1 \\
-a_{n} & -a_{n-1} & -a_{n-2} & \cdots & -a_{1}
\end{array}\right], \quad B=\left[\begin{array}{c}
0 \\
0 \\
\vdots \\
0 \\
1
\end{array}\right]
$$

Assume that all eigenvalues of $A$ are on the unit circle. Let $F(\varepsilon):(0,1] \rightarrow \mathbf{R}^{1 \times n}$ be the unique state feedback gain such that

$$
\lambda(A+B F(\varepsilon))=(1-\varepsilon) \lambda(A), \quad \varepsilon \in(0,1]
$$

Then, we have the following lemmas [17] on the properties of the resulting closed-loop system. Explicit construction of all the matrices involved in these lemmas can be found in [17]. 
Lemma 1. Consider the system (3) and let $F$ be as given by (5). Then, there exists a nonsingular transformation matrix $Q(\varepsilon) \in \mathbf{R}^{n \times n}$ such that

(6) $Q^{-1}(\varepsilon)(A+B F(\varepsilon)) Q(\varepsilon)=J(\varepsilon):=\operatorname{blkdiag}\left\{J_{-1}(\varepsilon), J_{+1}(\varepsilon), J_{1}(\varepsilon), \cdots, J_{l}(\varepsilon)\right\}$,

where

$$
\begin{gathered}
J_{-1}(\varepsilon)=\left[\begin{array}{rrrr}
-(1-\varepsilon) & 1 & & \\
& \ddots & \ddots & \\
& & -(1-\varepsilon) & 1 \\
& & & -(1-\varepsilon)
\end{array}\right]_{n_{-1} \times n_{-1}}, \\
J_{+1}(\varepsilon)=\left[\begin{array}{rrrr}
1-\varepsilon & 1 & & \\
& \ddots & \ddots & 1 \\
& & 1-\varepsilon & 1-\varepsilon
\end{array}\right]_{n_{+1} \times n_{+1}},
\end{gathered}
$$

and for each $i=1$ to $l$,

$$
J_{i}(\varepsilon)=\left[\begin{array}{rrrr}
J_{i}^{\star}(\varepsilon) & I_{2} & & \\
& \ddots & \ddots & \\
& & J_{i}^{\star}(\varepsilon) & I_{2} \\
& & & J_{i}^{\star}(\varepsilon)
\end{array}\right]_{2 n_{i} \times 2 n_{i}} \quad, J_{i}^{\star}(\varepsilon)=(1-\varepsilon)\left[\begin{array}{rr}
\alpha_{i} & \beta_{i} \\
-\beta_{i} & \alpha_{i}
\end{array}\right],
$$

with $\alpha_{i}^{2}+\beta_{i}^{2}=1$ for all $i=1$ to $l$ and $\alpha_{i} \neq \alpha_{j}$ for $i \neq j$.

Lemma 2. Consider the system (3) and let $F$ be as given by (5). Let $J(\varepsilon)$ be as given in Lemma 1. Let

$$
S(\varepsilon)=\operatorname{blkdiag}\left\{S_{-1}(\varepsilon), S_{+1}(\varepsilon), S_{1}(\varepsilon), S_{2}(\varepsilon), \cdots, S_{l}(\varepsilon)\right\},
$$

where $S_{-1}(\varepsilon)=\operatorname{diag}\left\{\varepsilon^{n_{-1}-1}, \varepsilon^{n_{-1}-2}, \cdots, \varepsilon, 1\right\}, S_{+1}(\varepsilon)=\operatorname{diag}\left\{\varepsilon^{n_{+1}-1}, \varepsilon^{n_{+1}-2}, \cdots, \varepsilon\right.$, $1\}$, and for each $i=1$ to $l, S_{i}(\varepsilon)=$ blkdiag $\left\{\varepsilon^{n_{i}-1} I_{2}, \varepsilon^{n_{i}-2} I_{2}, \cdots, \varepsilon I_{2}, I_{2}\right\}$.

Then,

1.

(11) $S(\varepsilon) J(\varepsilon) S^{-1}(\varepsilon)=\tilde{J}(\varepsilon):=\operatorname{blkdiag}\left\{\tilde{J}_{-1}(\varepsilon), \tilde{J}_{+1}(\varepsilon), \tilde{J}_{1}(\varepsilon), \cdots, \tilde{J}_{l}(\varepsilon)\right\}$,

where

$$
\tilde{J}_{-1}(\varepsilon)=\left[\begin{array}{rrrr}
-(1-\varepsilon) & \varepsilon & & \\
& \ddots & \ddots & \\
& & -(1-\varepsilon) & \varepsilon \\
& & & -(1-\varepsilon)
\end{array}\right]_{n_{-1} \times n_{-1}}
$$




$$
\tilde{J}_{+1}(\varepsilon)=\left[\begin{array}{rrrr}
(1-\varepsilon) & \varepsilon & & \\
& \ddots & \ddots & \\
& & (1-\varepsilon) & \varepsilon \\
& & & (1-\varepsilon)
\end{array}\right]_{n_{+1} \times n_{+1}},
$$

and for each $i=1$ to $l$,

$$
\tilde{J}_{i}(\varepsilon)=\left[\begin{array}{rrrr}
J_{i}^{\star}(\varepsilon) & \varepsilon I_{2} & & \\
& \ddots & \ddots & \\
& & J_{i}^{\star}(\varepsilon) & \varepsilon I_{2} \\
& & & J_{i}^{\star}(\varepsilon)
\end{array}\right]_{2 n_{i} \times 2 n_{i}}, J_{i}^{\star}(\varepsilon)=(1-\varepsilon)\left[\begin{array}{rr}
\alpha_{i} & \beta_{i} \\
-\beta_{i} & \alpha_{i}
\end{array}\right],
$$

with $\beta_{i}>0$ for all $i=1$ to $l$ and $\beta_{i} \neq \beta_{j}$ for $i \neq j$;

2. There exists an $\varepsilon^{*} \in(0,1]$ such that the unique positive definite solution $\tilde{P}(\varepsilon)$ to the Lyapunov equation

$$
\tilde{J}^{T}(\varepsilon) \tilde{P} \tilde{J}(\varepsilon)-\tilde{P}=-\varepsilon I
$$

is bounded over $\varepsilon \in\left(0, \varepsilon^{*}\right]$, i.e., there exist positive definite matrices $\tilde{P}_{1}$ and $\tilde{P}_{2}$ such that

$$
\tilde{P}_{1} \leq \tilde{P}(\varepsilon) \leq \tilde{P}_{2}, \quad \forall \varepsilon \in\left(0, \varepsilon^{*}\right]
$$

Lemma 3. Consider the system (3) and let $F$ be as given by (5). Let $Q(\varepsilon), l$, and $n_{i}$ for $i=0$ to $l$, be as defined in Lemma 1. Let the scaling matrix $S(\varepsilon)$ be as defined in Lemma 2. Then, there exist $\gamma, \alpha, \beta, \vartheta>0$, all independent of $\varepsilon$, such that, for all $\varepsilon \in(0,1]$,

$$
\begin{gathered}
|F(\varepsilon)| \leq \gamma \varepsilon, \\
\left|F(\varepsilon) Q(\varepsilon) S^{-1}(\varepsilon)\right| \leq \alpha \varepsilon, \\
\left|F(\varepsilon) A Q(\varepsilon) S^{-1}(\varepsilon)\right| \leq \beta \varepsilon, \\
|Q(\varepsilon)| \leq \vartheta,\left|Q^{-1}(\varepsilon)\right| \leq \vartheta .
\end{gathered}
$$

2.2. The Razumikhin Theorem. Consider the time delay system,

$$
\left\{\begin{aligned}
x(k+1) & =f\left(x(k), x_{k}\right), \quad k \geq 0, \\
x_{0}(s) & =\psi(s), \quad s \in[-r, 0] .
\end{aligned}\right.
$$


Let

$$
\mathcal{C}_{n, r}=\left\{\psi:\{-r,-r+1, \cdots,-1,0\} \longmapsto \mathbf{R}^{n}\right\}
$$

and for each $\psi \in \mathcal{C}_{n, r}$, we define its norm as

$$
\|\psi\|=\max _{s \in \mathcal{I}_{r}}|\psi(s)|
$$

where $|\cdot|$ is the Euclidean norm on $\mathbf{R}^{n}$ and $\mathcal{I}_{r}$ denotes the set of integers $\{-r,-r+$ $1, \cdots,-1,0\}$. We also use $x_{k} \in \mathcal{C}_{n, r}$ to denote the restriction of $x(k)$ to the interval $[k-r, k]$ translated to $[-r, 0]$, that is,

$$
x_{k}(s)=x(k+s), \quad s \in[-r, 0] .
$$

Also denote the solution of the difference equation (21) with the initial condition $x_{0} \in \mathcal{C}_{n, r}$ as $x\left(k, x_{0}\right)$.

DEFINITION 1. The zero solution $x(k) \equiv 0$ of (21) is said to be asymptotically stable if

1. for every $\delta>0$ there exists an $\epsilon=\epsilon(\delta)$ such that, for any $\psi \in \mathcal{B}(0, \epsilon):=$ $\left\{\psi \in \mathcal{C}_{n, r},\|\psi\| \leq \epsilon\right\}$, the solution $x(k, \psi)$ of (21) satisfies $x_{k} \in \mathcal{B}(0, \delta)$ for all $k \geq 0$

2. for every $\eta>0$ there exist a $K(\eta)$ and a $v_{0}>0$ independent of $\eta$ such that $\psi \in \mathcal{B}\left(0, v_{0}\right)$ implies that $\left\|x_{k}\right\|<\eta, \forall k \geq K(\eta)$.

If the system (21) is asymptotically and item 2 holds for every $\psi \in \mathcal{C}_{n, k}$, then it is globally asymptotically stable.

Clearly, if the system (21) is a linear system, then, asymptotic stability implies global asymptotic stability. The Razumikhin Theorem [7, 27] gives conditions under which $x(k) \equiv 0$ is asymptotically stable. Actually, more information about the invariant set and the regional stability is contained in the proof of this theorem in [7]. This additional information is incorporated in the following statement of the theorem.

Lemma 4 (Razumikhin Stability Theorem). Consider the delay difference equation (21). Suppose that $u, v, w$ and $p \in \mathbf{R}^{+} \longmapsto \mathbf{R}^{+}$are scalar, continuous and strictly increasing functions, with $u(0)=v(0)=0$ and $p(t)>t$ for $t>0$. If there is a continuous function $V: \mathbf{R}^{n} \longmapsto \mathbf{R}$ and a positive number $\rho$, such that for all

$$
x_{k} \in M_{V}(\rho):=\left\{\psi \in \mathcal{C}_{n, r}: V(\psi(s)) \leq \rho, \forall s \in[-r, 0]\right\}
$$

the following conditions hold,

1. $u(|x|) \leq V(x) \leq v(|x|)$,

2. $\Delta V(x(k))=V(x(k+1))-V(x(k)) \leq-w(|x(k)|)$, if $V(x(k+s))<p(V(x(k)))$, $\forall s \in[-r, 0]$, 
then the solution $x(k) \equiv 0$ of the equation (21) is asymptotically stable. Moreover, the set $M_{V}(\rho)$ is an invariant set inside the domain of attraction. If items 1 and 2 hold for all $x \in \mathbf{R}^{n}$ and $u(t) \rightarrow \infty$ as $t \rightarrow \infty$, then, the solution $x(k) \equiv 0$ is globally asymptotically stable.

3. Stabilization by Linear State Feedback. For the system (1) with all eigenvalues of $A$ on or inside the unit circle, we construct two families of linear state feedback laws as follows.

State Feedback Design:

Step 1. Find nonsingular transformation matrices $T_{\mathrm{S}}$ and $T_{\mathrm{I}}$ such that the pair $(A, B)$ is transformed into the following block diagonal control canonical form,

$$
\begin{aligned}
T_{\mathrm{S}}^{-1} A T_{\mathrm{S}} & =\left[\begin{array}{ccccc}
A_{1} & 0 & \cdots & 0 & 0 \\
0 & A_{2} & \cdots & 0 & 0 \\
\vdots & \vdots & \ddots & \vdots & \vdots \\
0 & 0 & \cdots & A_{l} & 0 \\
0 & 0 & \cdots & 0 & A_{0}
\end{array}\right], \\
T_{\mathrm{S}}^{-1} B T_{\mathrm{I}} & =\left[\begin{array}{ccccc}
B_{1} & B_{12} & \cdots & B_{1 l} & * \\
0 & B_{2} & \cdots & B_{2 l} & * \\
\vdots & \vdots & \ddots & \vdots & \vdots \\
0 & 0 & \cdots & B_{l} & * \\
B_{01} & B_{02} & \cdots & B_{0 l} & *
\end{array}\right],
\end{aligned}
$$

where $A_{0}$ contains all the eigenvalues of $A$ that are strictly inside the unit circle, for each $i=1$ to $l$, all eigenvalues of $A_{i}$ are on the unit circle and hence $\left(A_{i}, B_{i}\right)$ is controllable and is given by,

$$
A_{i}=\left[\begin{array}{ccccc}
0 & 1 & 0 & \cdots & 0 \\
0 & 0 & 1 & \cdots & 0 \\
\vdots & \vdots & \vdots & \ddots & \vdots \\
0 & 0 & 0 & \cdots & 1 \\
-a_{i n_{i}} & -a_{i\left(n_{i}-1\right)} & -a_{i\left(n_{i}-2\right)} & \cdots & -a_{i 1}
\end{array}\right], \quad B_{i}=\left[\begin{array}{c}
0 \\
0 \\
\vdots \\
0 \\
1
\end{array}\right]
$$

and finally, *'s represent submatrices of less interest.

We note that the existence of the above canonical form was shown in [21].

The software realization can be found in [11].

Step 2. For each $\left(A_{i}, B_{i}\right)$, let $F_{i}(\varepsilon) \in \mathbf{R}^{1 \times n_{i}}$ be the state feedback gain such that

$$
\lambda\left(A_{i}+B_{i} F_{i}(\varepsilon)\right)=(1-\varepsilon) \lambda\left(A_{i}\right), \quad \varepsilon \in(0,1] .
$$

Note that $F_{i}(\varepsilon)$ is unique. 
Step 3. Construct two families of low gain state feedback laws as

$$
u(k)=F(\varepsilon) A^{r} x(k),
$$

and

$$
u(k)=F(\varepsilon) x(k),
$$

where the low gain matrix $F(\varepsilon)$ is given by

$$
F(\varepsilon)=T_{\mathrm{I}}\left[\begin{array}{cccccc}
F_{1}(\varepsilon) & 0 & \cdots & 0 & 0 & 0 \\
0 & F_{2}(\varepsilon) & \cdots & 0 & 0 & 0 \\
\vdots & \vdots & \ddots & \vdots & \vdots & \vdots \\
0 & 0 & \cdots & F_{l-1}(\varepsilon) & 0 & 0 \\
0 & 0 & \cdots & 0 & F_{l}(\varepsilon) & 0 \\
0 & 0 & \cdots & 0 & 0 & 0
\end{array}\right] T_{\mathrm{S}}^{-1} .
$$

The first class of low gain feedback laws (25) uses the knowledge of the time delay $r$, while the second family (26) does not require the knowledge of $r$. The theorem below establishes that a linear state feedback law from (25) asymptotically stabilizes the system (1) as long as all eigenvalues of $A$ are on or inside the unit circle.

THEOREM 1. Consider the closed-loop system comprising of the system (1) and the linear state feedback law (25). Let all eigenvalues of $A$ be on or inside the unit circle. Then, for any given arbitrarily large $r \geq 0$, there exists an $\varepsilon^{*}>0$, such that, for each $\varepsilon \in\left(0, \varepsilon^{*}\right]$, the closed-loop system is asymptotically stable.

Proof. Without loss of generality, assume that the pair $(A, B)$ are already in the form of (22)-(23). Under the state feedback law (25), the closed-loop system is given by

$$
x(k+1)=A x(k)+B F(\varepsilon) A^{r} x(k-r)
$$

from which we have

$$
A^{r} x(k-r)=x(k)-\sum_{s=0}^{r-1} A^{r-s-1} B F(\varepsilon) A^{r} x(k+s-2 r) .
$$

Substitution of (29) in (28) results in

$$
x(k+1)=(A+B F(\varepsilon)) x(k)-B F(\varepsilon) \sum_{s=0}^{r-1} A^{r-s-1} B F(\varepsilon) A^{r} x(k+s-2 r) .
$$

Partitioning the state $x$ according to the structure of (22)-(23),

$$
x=\left[\begin{array}{lllll}
x_{1}^{\mathrm{T}} & x_{2}^{\mathrm{T}} & \cdots & x_{l}^{\mathrm{T}} & x_{0}^{\mathrm{T}}
\end{array}\right]^{\mathrm{T}}, \quad x_{i} \in \mathbf{R}^{n_{i}}, i \in[1, l],
$$


we rewrite the state equation (30) as follows

$$
\left\{\begin{aligned}
x_{1}(k+1)= & \left(A_{1}+B_{1} F_{1}(\varepsilon)\right) x_{1}(k)+\sum_{j=2}^{l} B_{1 j} F_{j}(\varepsilon) x_{j}(k) \\
& -B_{\mathrm{R} 1} F(\varepsilon) \sum_{s=0}^{r-1} A^{r-s-1} B F(\varepsilon) A^{r} x(k+s-2 r), \\
x_{2}(k+1)= & \left(A_{2}+B_{2} F_{2}(\varepsilon)\right) x_{2}(k)+\sum_{j=3}^{l} B_{2 j} F_{j}(\varepsilon) x_{j}(k) \\
& -B_{\mathrm{R} 2} F(\varepsilon) \sum_{s=0}^{r-1} A^{r-s-1} B F(\varepsilon) A^{r} x(k+s-2 r), \\
\vdots & \left(A_{l}+B_{l} F_{l}(\varepsilon)\right) x_{l}(k) \\
& -B_{\mathrm{R} l} F(\varepsilon) \sum_{s=0}^{r-1} A^{r-s-1} B F(\varepsilon) A^{r} x(k+s-2 r), \\
x_{l}(k+1) & A_{0} x_{0}(k)+\sum_{j=1}^{l} B_{0 j} F_{j}(\varepsilon) x_{j}(k) \\
& -B_{\mathrm{R} 0} F(\varepsilon) \sum_{s=0}^{r-1} A^{r-s-1} B F(\varepsilon) A^{r} x(k+s-2 r), \\
x_{0}(k+1) &
\end{aligned}\right.
$$

where for each $i=1$ to $l, B_{\mathrm{R} i}$ is the $i$ th row of the right hand side of (23) and $B_{\mathrm{R} 0}$ is the last row.

Now for each $i=1$ to $l$, let $Q_{i}(\varepsilon), S_{i}(\varepsilon), \tilde{J}_{i}(\varepsilon), \tilde{P}_{i}, \gamma_{i}, \alpha_{i}, \beta_{i}$, and $\vartheta_{i}$ be the matrices $Q(\varepsilon), S(\varepsilon), \tilde{J}(\varepsilon)$ and $\tilde{P}$ and the constants $\gamma, \alpha, \beta$ and $\vartheta$ as defined in Lemmas 1-3, but for the triple $\left(A_{i}, B_{i}, F_{i}(\varepsilon)\right)$. Define a state transformation as,

$$
\tilde{x}=\left[\tilde{x}_{1}^{\mathrm{T}}, \tilde{x}_{2}^{\mathrm{T}}, \cdots, \tilde{x}_{l}^{\mathrm{T}}, \tilde{x}_{0}^{\mathrm{T}}\right]^{\mathrm{T}},
$$

where $\tilde{x}_{0}=x_{0}$, and, for each $i=1$ to $l, \tilde{x}_{i}=S_{i}(\varepsilon) Q_{i}^{-1}(\varepsilon) x_{i}$.

It follows from Lemmas 1 and 2 that, under this state transformation, the state 
equation (31) can be written as

(33)

$$
\left\{\begin{aligned}
\tilde{x}_{1}(k+1) & =\tilde{J}_{1}(\varepsilon) \tilde{x}_{1}(k)+\sum_{j=2}^{l} S_{1}(\varepsilon) Q_{1}^{-1}(\varepsilon) B_{1 j} F_{j}(\varepsilon) Q_{j}(\varepsilon) S_{j}^{-1}(\varepsilon) \tilde{x}_{j}(k) \\
& -S_{1}(\varepsilon) Q_{1}^{-1}(\varepsilon) B_{\mathrm{R} 1} F(\varepsilon) \sum_{s=0}^{r-1} A^{r-s-1} B F(\varepsilon) A^{r} Q(\varepsilon) S^{-1}(\varepsilon) \tilde{x}(k+s-2 r), \\
\tilde{x}_{2}(k+1) & =\tilde{J}_{2}(\varepsilon) \tilde{x}_{2}(k)+\sum_{j=3}^{l} S_{2}(\varepsilon) Q_{2}(\varepsilon) B_{2 j} F_{j}(\varepsilon) Q_{j}(\varepsilon) S_{j}^{-1}(\varepsilon) \tilde{x}_{j}(k) \\
& -S_{2}(\varepsilon) Q_{2}^{-1}(\varepsilon) B_{\mathrm{R} 2} F(\varepsilon) \sum_{s=0}^{r-1} A^{r-s-1} B F(\varepsilon) A^{r} Q(\varepsilon) S^{-1}(\varepsilon) \tilde{x}(k+s-2 r), \\
& \vdots \\
& -S_{l}(\varepsilon) Q_{l}^{-1}(\varepsilon) B_{\mathrm{R} l} F(\varepsilon) \sum_{s=0}^{r-1} A^{r-s-1} B F(\varepsilon) A^{r} Q(\varepsilon) S^{-1}(\varepsilon) \tilde{x}(k+s-2 r), \\
\tilde{x}_{l}(k+1) & \tilde{J}_{l}(\varepsilon) \tilde{x}_{l}(k) \\
& -B_{\mathrm{R} 0} F(\varepsilon) \sum_{s=0}^{r-1} A^{r-s-1} B F(\varepsilon) A^{r} Q(\varepsilon) S^{-1}(\varepsilon) \tilde{x}(k+s-2 r)
\end{aligned}\right.
$$

where

$$
Q(\varepsilon)=\operatorname{blkdiag}\left\{Q_{1}(\varepsilon), Q_{2}(\varepsilon), \cdots, Q_{l}(\varepsilon), I\right\}
$$

and

$$
S(\varepsilon)=\operatorname{blkdiag}\left\{S_{1}(\varepsilon), S_{2}(\varepsilon), \cdots, S_{l}(\varepsilon), I\right\} .
$$

Let us choose a Lyapunov function

$$
V(\tilde{x})=\sum_{i=1}^{l} \kappa^{i} \tilde{x}_{i}^{\mathrm{T}} \tilde{P}_{i}(\varepsilon) \tilde{x}_{i}+\tilde{x}_{0}^{\mathrm{T}} \tilde{P}_{0} \tilde{x}_{0}:=\tilde{x}^{\mathrm{T}} \tilde{P}(\varepsilon) \tilde{x},
$$

where $\tilde{P}_{0}>0$ is such that

$$
A_{0}^{\mathrm{T}} \tilde{P}_{0} A_{0}-\tilde{P}_{0}=-I
$$

$\kappa>0$ is a constant whose value is to be determined later, and

$$
\tilde{P}(\varepsilon)=\operatorname{blkdiag}\left\{\kappa \tilde{P}_{1}(\varepsilon), \kappa^{2} \tilde{P}_{2}(\varepsilon), \cdots, \kappa^{l} \tilde{P}_{l}(\varepsilon), \tilde{P}_{0}\right\} .
$$

The existence of such a $\tilde{P}_{0}$ is due to the fact that $A_{0}$ is asymptotically stable. The difference of $V$ along the trajectories of the closed-loop system (33) can be evaluated 
as follows,

$$
\begin{aligned}
& \Delta V(\tilde{x}(k))=\sum_{i=1}^{l}\left[\kappa^{i} \tilde{x}_{i}^{\mathrm{T}}(k)\left(\tilde{J}_{i}^{\mathrm{T}}(\varepsilon) \tilde{P}_{i}(\varepsilon) \tilde{J}_{i}(\varepsilon)-\tilde{P}_{i}\right) \tilde{x}_{i}(k)\right. \\
& +2 \sum_{j=i+1}^{l} \kappa^{i} \tilde{x}_{i}^{\mathrm{T}}(k) \tilde{J}_{i}^{\mathrm{T}}(\varepsilon) \tilde{P}_{i}(\varepsilon) S_{i}(\varepsilon) Q_{i}^{-1}(\varepsilon) B_{i j} F_{j}(\varepsilon) Q_{j}(\varepsilon) S_{j}^{-1}(\varepsilon) \tilde{x}_{j}(k) \\
& -2 \kappa^{i}\left[\sum_{j=i+1}^{l} S_{i}(\varepsilon) Q_{i}^{-1}(\varepsilon) B_{i j} F_{j}(\varepsilon) Q_{j}(\varepsilon) S_{j}^{-1}(\varepsilon) \tilde{x}_{j}(k)\right]^{\mathrm{T}} \tilde{P}_{i}(\varepsilon) \\
& \times S_{i}(\varepsilon) Q_{i}^{-1}(\varepsilon) B_{\mathrm{R} i} F(\varepsilon) \sum_{s=0}^{r-1} A^{r-s-1} B F(\varepsilon) A^{r} Q(\varepsilon) S^{-1}(\varepsilon) \tilde{x}(k+s-2 r) \\
& +k^{i}\left[\sum_{j=i+1}^{l} S_{i}(\varepsilon) Q_{i}^{-1}(\varepsilon) B_{i j} F_{j}(\varepsilon) Q_{j}(\varepsilon) S_{j}^{-1}(\varepsilon) \tilde{x}_{j}(k)\right]^{\mathrm{T}} \tilde{P}_{i}(\varepsilon) \\
& \left.\times\left[\sum_{j=i+1}^{l} S_{i}(\varepsilon) Q_{i}^{-1}(\varepsilon) B_{i j} F_{j}(\varepsilon) Q_{j}(\varepsilon) S_{j}^{-1}(\varepsilon) \tilde{x}_{j}(k)\right]\right] \\
& -\tilde{x}_{0}^{\mathrm{T}}(k)\left(A_{0}^{\mathrm{T}} \tilde{P}_{0} A_{0}-\tilde{P}_{0}\right) \tilde{x}_{0}(k)+2 \sum_{j=1}^{l} \tilde{x}_{0}^{\mathrm{T}}(k) A_{0}^{\mathrm{T}} \tilde{P}_{0} B_{0 j} F_{j}(\varepsilon) Q_{j}(\varepsilon) S_{j}^{-1}(\varepsilon) \tilde{x}_{j}(k) \\
& +\left[\sum_{j=1}^{l} B_{0 j} F_{j}(\varepsilon) Q_{j}(\varepsilon) S_{j}^{-1}(\varepsilon) \tilde{x}_{j}(k)\right]^{\mathrm{T}} P_{0}\left[\sum_{j=1}^{l} B_{0 j} F_{j}(\varepsilon) Q_{j}(\varepsilon) S_{j}^{-1}(\varepsilon) \tilde{x}_{j}(k)\right] \\
& -2\left[\sum_{i=1}^{l} \kappa^{i} \tilde{x}_{i}^{\mathrm{T}}(k) \tilde{J}_{i}^{\mathrm{T}}(\varepsilon) \tilde{P}_{i}(\varepsilon) S_{i}(\varepsilon) Q_{i}^{-1}(\varepsilon) B_{\mathrm{R} i}+\tilde{x}_{0}^{\mathrm{T}} A_{0}^{\mathrm{T}} \tilde{P}_{0} B_{\mathrm{R} 0}\right] F(\varepsilon) \\
& \times \sum_{s=0}^{r-1} A^{r-s-1} B F(\varepsilon) A^{r} Q(\varepsilon) S^{-1}(\varepsilon) \tilde{x}(k+s-2 r) \\
& +\left[\sum_{s=0}^{r-1} A^{r-s-1} B F(\varepsilon) A^{r} Q(\varepsilon) S^{-1}(\varepsilon) \tilde{x}(k+s-2 r)\right]^{\mathrm{T}} \\
& \times\left[\sum_{i=1}^{l} \kappa^{i} F^{\mathrm{T}}(\varepsilon) B_{\mathrm{R} i}^{\mathrm{T}}\left(Q_{i}^{-1}\right)^{\mathrm{T}} S_{i}^{\mathrm{T}}(\varepsilon) \tilde{P}_{i}(\varepsilon) S_{i}(\varepsilon) Q_{i}^{-1}(\varepsilon) B_{\mathrm{R} i} F(\varepsilon)\right. \\
& \left.+F^{\mathrm{T}}(\varepsilon) B_{\mathrm{R} 0}^{\mathrm{T}} \tilde{P}_{0} B_{\mathrm{R} 0} F(\varepsilon)\right] \\
& \times\left[\sum_{s=0}^{r-1} A^{r-s-1} B F(\varepsilon) A^{r} Q(\varepsilon) S^{-1}(\varepsilon) \tilde{x}(k+s-2 r)\right] .
\end{aligned}
$$

Recall that $\tilde{J}^{\mathrm{T}}(\varepsilon) \tilde{P}_{i}(\varepsilon) \tilde{J}_{i}(\varepsilon)-\tilde{P}_{i}=-\varepsilon I$ and $A_{0}^{\mathrm{T}} \tilde{P}_{0} A_{0}-\tilde{P}_{0}=-I$. Also, it follows from Lemma 3 that the matrices defining the remaining $\left(\tilde{x}_{i}(k), \tilde{x}_{j}(k)\right)$ terms, other than the term defined by $A_{0}^{\mathrm{T}} \tilde{P}_{0} A_{0}-\tilde{P}_{0}$, are all of order $\varepsilon$ or order $\varepsilon^{2}$. It is then 
straightforward to verify that there exist a constant $\kappa>0$ and an $\varepsilon_{1}^{*} \in(0,1]$ such that,

$$
\begin{aligned}
& \Delta V(\tilde{x}(k)) \leq-\frac{\varepsilon}{2} \tilde{x}^{\mathrm{T}}(k) \tilde{x}(k) \\
& -2\left[\tilde{x}_{0}^{\mathrm{T}} A_{0}^{\mathrm{T}} \tilde{P}_{0} B_{\mathrm{R} 0}+\sum_{i=1}^{l} \kappa^{i}\left[\tilde{x}_{i}^{\mathrm{T}}(k) \tilde{J}_{i}^{\mathrm{T}}(\varepsilon) \tilde{P}_{i}(\varepsilon) S_{i}(\varepsilon) Q_{i}^{-1}(\varepsilon) B_{\mathrm{R} i}\right.\right. \\
& +\left[\sum_{j=i+1}^{l} S_{i}(\varepsilon) Q^{-1}(\varepsilon) B_{i j} F_{j}(\varepsilon) Q_{j}(\varepsilon) S_{j}^{-1}(\varepsilon) \tilde{x}_{j}(k)\right]^{\mathrm{T}} \\
& \left.\left.\times \tilde{P}_{i}(\varepsilon) S_{i}(\varepsilon) Q_{i}^{-1}(\varepsilon) B_{\mathrm{R} i}\right]\right] F(\varepsilon) \\
& \times \sum_{s=0}^{r-1} A^{r-s-1} B F(\varepsilon) A^{r} Q(\varepsilon) S^{-1}(\varepsilon) \tilde{x}(k+s-2 r) \\
& +\left[\sum_{s=0}^{r-1} A^{r-s-1} B F(\varepsilon) A^{r} Q(\varepsilon) S^{-1}(\varepsilon) \tilde{x}(k+s-2 r)\right]^{\mathrm{T}} \\
& \times\left[\sum_{i=1}^{l} \kappa^{i} F^{\mathrm{T}}(\varepsilon) B_{\mathrm{R} i}^{\mathrm{T}}\left(Q_{i}^{-1}(\varepsilon)\right)^{\mathrm{T}} S_{i}^{\mathrm{T}}(\varepsilon) S_{i}(\varepsilon) Q_{i}^{-1}(\varepsilon) B_{\mathrm{R} i} F(\varepsilon)\right. \\
& \left.+F^{\mathrm{T}}(\varepsilon) B_{\mathrm{R} 0}^{\mathrm{T}} B_{\mathrm{R} 0} F(\varepsilon)\right] \\
& \times\left[\sum_{s=0}^{r-1} A^{r-s-1} B F(\varepsilon) A^{r} Q(\varepsilon) S^{-1}(\varepsilon) \tilde{x}(k+s-2 r)\right], \quad \varepsilon \in\left(0, \varepsilon_{1}^{*}\right] .
\end{aligned}
$$

Recalling the special structure of $\tilde{J}_{i}(\varepsilon)$ and Lemma 3 , we have

$$
\begin{aligned}
\left|S(\varepsilon) Q^{-1}(\varepsilon) A Q(\varepsilon) S^{-1}(\varepsilon)\right|=\sum_{i=1}^{l}\left|S_{i}(\varepsilon) Q_{i}^{-1}(\varepsilon) A_{i} Q_{i}(\varepsilon) S_{i}^{-1}(\varepsilon)\right|+\left|A_{0}\right| \\
=\sum_{i=1}^{l} \mid S_{i}(\varepsilon) Q_{i}^{-1}(\varepsilon)\left(A_{i}+B_{i} F_{i}(\varepsilon)\right) Q(\varepsilon) S_{i}^{-1}(\varepsilon) \\
\quad-S_{i}(\varepsilon) Q_{i}^{-1}(\varepsilon) B_{i} F_{i}(\varepsilon) Q_{i}(\varepsilon) S_{i}^{-1}(\varepsilon)|+| A_{0} \mid \\
\leq \sum_{i=1}^{l}\left(\left|\tilde{J}_{i}(\varepsilon)\right|+\varepsilon \alpha_{i} \vartheta_{i}\left|B_{i}\right|\right)+\left|A_{0}\right| \\
\leq \delta, \quad \varepsilon \in\left(0, \varepsilon_{1}^{*}\right] .
\end{aligned}
$$

Hence, we have

$$
\begin{aligned}
& \left|S(\varepsilon) Q^{-1}(\varepsilon) A^{i} Q(\varepsilon) S^{-1}(\varepsilon)\right| \\
= & \left|S(\varepsilon) Q^{-1}(\varepsilon) A Q(\varepsilon) S^{-1}(\varepsilon) S(\varepsilon) Q^{-1}(\varepsilon) A \cdots A Q(\varepsilon) S^{-1}(\varepsilon)\right| \leq \delta^{i},
\end{aligned}
$$


and,

$$
\begin{aligned}
\mid F(\varepsilon) & A^{r} Q(\varepsilon) S^{-1}(\varepsilon)|=| F(\varepsilon) Q(\varepsilon) S^{-1}(\varepsilon) S(\varepsilon) Q^{-1}(\varepsilon) A^{r} Q(\varepsilon) S^{-1}(\varepsilon) \mid \\
\leq & \left|F(\varepsilon) Q(\varepsilon) S^{-1}(\varepsilon)\right|\left|S(\varepsilon) Q^{-1}(\varepsilon) A^{r} Q(\varepsilon) S^{-1}(\varepsilon)\right| \\
\leq & {\left[\sum_{i=1}^{l}\left|F_{i}(\varepsilon) Q_{i}(\varepsilon) S_{i}^{-1}(\varepsilon)\right|\right] \delta^{r} } \\
\leq & \varepsilon\left[\sum_{i=1}^{l} \alpha_{i}\right] \delta^{r} .
\end{aligned}
$$

Consequently, the inequality (37) can be continued as follows,

$$
\begin{aligned}
\Delta V(\tilde{x}(k)) \leq & -\frac{\varepsilon}{2} \lambda_{\max }^{-1}(\tilde{P}(\varepsilon)) V(\tilde{x}(k)) \\
& +\varepsilon^{2} \varpi_{1}(r) V^{\frac{1}{2}}(\tilde{x}(k)) \sum_{s=0}^{r-1}|A|^{r-s-1} V^{\frac{1}{2}}(\tilde{x}(k+s-2 r)), \\
& +\varepsilon^{4} \varpi_{2}(r)\left[\sum_{s=0}^{r-1}|A|^{r-s-1} V^{\frac{1}{2}}(\tilde{x}(k+s-2 r))\right]^{2}, \quad \varepsilon \in\left(0, \varepsilon_{1}^{*}\right],
\end{aligned}
$$

for some $\varpi_{1}(r), \varpi_{2}(r)>0$, both independent of $\varepsilon$. In arriving at the above inequality, we have used the inequality

$$
V(\tilde{x}(k))=\tilde{x}^{\mathrm{T}}(k) \tilde{P}(\varepsilon) \tilde{x}(k) \leq \lambda_{\max }(\tilde{P}(\varepsilon)) \tilde{x}^{\mathrm{T}}(k) \tilde{x}(k) .
$$

Now, let $\eta>1$ be any constant. If $V(\tilde{x}(k+s))<\eta V(\tilde{x}(k)), \forall s \in[-r, 0]$, then,

$$
\begin{aligned}
\Delta V(\tilde{x}(k)) \leq & -\frac{\varepsilon}{2} \lambda_{\max }^{-1}(\tilde{P}(\varepsilon)) V(\tilde{x}(k))+\varepsilon^{2} \varpi_{1}(r) \eta\left[\sum_{s=0}^{r-1}|A|^{r-s-1}\right] V(\tilde{x}(k)) \\
& +\varepsilon^{4} \varpi_{2}(r) \eta^{2}\left[\sum_{s=0}^{r-1}|A|^{r-s-1}\right]^{2} V(\tilde{x}(k)) \\
= & -\varepsilon\left[\frac{1}{2} \lambda_{\max }^{-1}(\tilde{P}(\varepsilon))-\varepsilon \varpi_{1}(r) \eta\left[\sum_{s=0}^{r-1}|A|^{r-s-1}\right]\right. \\
& \left.-\varepsilon^{3} \varpi_{2}(r) \eta^{2}\left[\sum_{s=0}^{r-1}|A|^{r-s-1}\right]^{2}\right] V(\tilde{x}(k)), \quad \varepsilon \in\left(0, \varepsilon_{1}^{*}\right],
\end{aligned}
$$

where the following estimate was used,

$$
V^{\frac{1}{2}}(\tilde{x}(k+s-2 r)) \leq \sqrt{\eta} V^{\frac{1}{2}}(\tilde{x}(k-r)) \leq \eta V^{\frac{1}{2}}(\tilde{x}(k)), \quad \forall s \in[-r, 0] .
$$

We recall that, by the definition of $\tilde{P}(\varepsilon)$ in $(35)$ and because of $(16), \lambda_{\max }(\tilde{P}(\varepsilon))$, as a function of $\varepsilon$, is bounded from below by a positive scalar independent of $\varepsilon$. It is 
clear that, for any given $r \geq 0$ and $\eta>0$, there exists an $\varepsilon^{*} \in\left(0, \varepsilon_{1}^{*}\right]$, such that, for all $\varepsilon \in\left(0, \varepsilon^{*}\right]$,

$$
\Delta V(\tilde{x}(k)) \leq-\mu(\varepsilon) V(\tilde{x}(k)), \quad \text { if } V(\tilde{x}(k+s))<\eta V(\tilde{x}(k)), \quad \forall s \in[-r, 0],
$$

for some positive scalar $\mu(\varepsilon)$. It thus follows from Lemma 4 that the closed-loop system (28) is asymptotically stable.

Theorem 1 involves the linear state feedback law (25), whose gain matrix depends explicitly on the value of $r$. While for some special cases, delay independent feedback laws might achieve stabilization, the knowledge of the delay $r$ is in general necessary. To see this, consider the simple system,

$$
x(k+1)=-x(k)+u(k-r), \quad x \in \mathbf{R},
$$

which has a single open loop pole located on the unit circle at $z=-1$. Let us consider a delay independent feedback law

$$
u(k)=\alpha x(k) .
$$

Under this feedback law, the closed-loop system is given by

$$
x(k+1)=-x(k)+\alpha x(k-r),
$$

which has the following characteristic equation

$$
z^{r+1}+z^{r}-\alpha=0
$$

Using the Jury table, it is straightforward to verified that, when $r$ is an odd number, a necessary condition for the system to be stable is $\alpha<0$, and for an even $r$, a necessary condition for the system to be stable is $\alpha>0$. This clearly shows that the knowledge of the delay $r$ is indispensable in the construction of stabilizing feedback laws.

Given this necessary condition, let us construct a state feedback law of the form (25) as follows

$$
u(k)=F(\varepsilon) A^{r} x(k)=(-1)^{r} \varepsilon x(k), \quad \varepsilon>0,
$$

which results in the following closed-loop system characteristic equation

$$
z^{r+1}+z^{r}-(-1)^{r} \varepsilon=0 .
$$

Using root locus arguments, it can be verified that the closed-loop system is indeed asymptotically stable for sufficiently small value of $\varepsilon$, as Theorem 1 indicates. The root locus arguments also justifies the use of low gain feedback. 
The following theorem shows that if all eigenvalues of $A$ are either at $z=1$ or strictly inside the unit circle, then the system (1) can indeed be asymptotically stabilized by a linear state feedback law (26), whose gain does not depend on the value of $r$. The intuition to this intricate difference between the poles at $z=1$ and the poles at other locations on the unit circle is the following. Under the low gain feedback, open loop poles at $z=1$ are relocated to $1-\varepsilon$. As a result, the closed-loop dynamics corresponding to these poles, and hence the control input, evolves slowly and the effect of the delay is small. On the other hand, under the low gain feedback, a pole at $z=-1$ or a conjugate pair of poles at other locations on the unit circle are shrunk toward the origin and relocated on the circle with a radius of $1-\varepsilon$. Even though the norm of the two states associated with these two poles decays slowly, the states, and hence the control input, oscillate at a constant frequency no matter how small $\varepsilon$ is. That is, the states oscillate and decay to zero within the envelope defined by $\pm(1-\varepsilon)^{k}$. Thus, the factor $A^{r}$ in the feedback gain is used to account for the effect of the delay in control input. This intricate difference between the oscillating nature of the closed-loop state trajectories resulting from an open loop pole at $z=-1$ or from pure imaginary open loop poles and the non-oscillating nature of the state trajectories resulting from open loop poles at $z=1$ can be easily observed in the simulation results presented in Section 6 .

TheOrem 2. Consider the closed-loop system comprising of the system (1) and the linear state feedback law (26). Let all eigenvalues of $A$ be either at $z=1$ or strictly inside the unit circle. Then, for any given arbitrarily large $r \geq 0$, there exists an $\varepsilon^{*}>0$, such that, for each $\varepsilon \in\left(0, \varepsilon^{*}\right]$, the closed-loop system is asymptotically stable.

Proof. Under the linear state feedback law (26), the closed-loop system is given by,

$$
\begin{aligned}
x(k+1) & =A x(k)+B F(\varepsilon) x(k-r) \\
& =(A+B F(\varepsilon)) x(k)+B F(\varepsilon)(x(k-r)-x(k)) .
\end{aligned}
$$

Without loss of generality, assume that the pair $(A, B)$ are already in the form of (22)-(23). Partitioning the state $x$ according to the structure of (22)-(23),

$$
x=\left[\begin{array}{lllll}
x_{1}^{\mathrm{T}} & x_{2}^{\mathrm{T}} & \cdots & x_{l}^{\mathrm{T}} & x_{0}^{\mathrm{T}}
\end{array}\right]^{\mathrm{T}}, \quad x_{i} \in \mathbf{R}^{n_{i}}, i \in[1, l],
$$


we rewrite the state equation (44) as follows

$$
\left\{\begin{aligned}
x_{1}(k+1)= & \left(A_{1}+B_{1} F_{1}(\varepsilon)\right) x_{1}(k) \\
& +\sum_{j=2}^{l} B_{1 j} F_{j}(\varepsilon) x_{j}(k)+B_{\mathrm{R} 1} F(\varepsilon)(x(k-r)-x(k)), \\
x_{2}(k+1)= & \left(A_{2}+B_{2} F_{2}(\varepsilon)\right) x_{2}(k) \\
& +\sum_{j=3}^{l} B_{2 j} F_{j}(\varepsilon) x_{j}(k)+B_{\mathrm{R} 2} F(\varepsilon)(x(k-r)-x(k)), \\
\vdots & \left(A_{l}+B_{l} F_{l}(\varepsilon)\right) x_{l}(k)+B_{\mathrm{R} l} F(\varepsilon)(x(k-r)-x(k)), \\
x_{l}(k+1)= & A_{0} x_{0}(k)+\sum_{j=1}^{l} B_{0 j} F_{j}(\varepsilon) x_{j}(k)+B_{\mathrm{R} 0} F(\varepsilon)(x(k-r)-x(k)), \\
x_{0}(k+1) &
\end{aligned}\right.
$$

where for each $i=1$ to $l, B_{\mathrm{R} i}$ is the $i$ th row of the right hand side of (23) and $B_{\mathrm{R} 0}$ is the last row.

For each $i=1$ to $l$, let $Q_{i}(\varepsilon), S_{i}(\varepsilon), \tilde{J}_{i}(\varepsilon), \tilde{P}_{i}, \gamma_{i}, \alpha_{i}, \beta_{i}$, and $\vartheta_{i}$ be the matrices $Q(\varepsilon), S(\varepsilon), \tilde{J}(\varepsilon)$ and $\tilde{P}$ and the constants $\gamma, \alpha, \beta$ and $\vartheta$ as defined in Lemmas 1-3, but for the triple $\left(A_{i}, B_{i}, F_{i}(\varepsilon)\right)$. Define a state transformation as,

$$
\tilde{x}=\left[\tilde{x}_{1}^{\mathrm{T}}, \tilde{x}_{2}^{\mathrm{T}}, \cdots, \tilde{x}_{l}^{\mathrm{T}}, \tilde{x}_{0}^{\mathrm{T}}\right]^{\mathrm{T}},
$$

where $\tilde{x}_{0}=x_{0}$, and, for each $i=1$ to $l, \tilde{x}_{i}=S_{i}(\varepsilon) Q_{i}^{-1}(\varepsilon) x_{i}$.

It follows from Lemmas 1 and 2 that, under this state transformation, the state equation (45) can be written as

$$
\left\{\begin{aligned}
\tilde{x}_{1}(k+1)= & \tilde{J}_{1}(\varepsilon) \tilde{x}_{1}(k)+\sum_{j=2}^{l} S_{1}(\varepsilon) Q_{1}^{-1}(\varepsilon) B_{1 j} F_{j}(\varepsilon) Q_{j}(\varepsilon) S_{j}^{-1}(\varepsilon) \tilde{x}_{j}(k) \\
& +S_{1}(\varepsilon) Q_{1}^{-1}(\varepsilon) B_{\mathrm{R} 1} F(\varepsilon) Q(\varepsilon) S^{-1}(\varepsilon)(\tilde{x}(k-r)-\tilde{x}(k)), \\
\tilde{x}_{2}(k+1)= & \tilde{J}_{2}(\varepsilon) \tilde{x}_{2}(k)+\sum_{j=3}^{l} S_{2}(\varepsilon) Q_{2}(\varepsilon) B_{2 j} F_{j}(\varepsilon) Q_{j}(\varepsilon) S_{j}^{-1}(\varepsilon) \tilde{x}_{j}(k) \\
& +S_{2}(\varepsilon) Q_{2}^{-1}(\varepsilon) B_{\mathrm{R} 2} F(\varepsilon) Q(\varepsilon) S^{-1}(\varepsilon)(\tilde{x}(k-r)-\tilde{x}(k)), \\
\vdots & \tilde{J}_{l}(\varepsilon) \tilde{x}_{l}(k)+S_{l}(\varepsilon) Q_{l}^{-1}(\varepsilon) B_{\mathrm{R} l} F(\varepsilon) Q(\varepsilon) S^{-1}(\varepsilon)(\tilde{x}(k-r)-\tilde{x}(k)), \\
\tilde{x}_{l}(k+1) & A_{0} \tilde{x}_{0}(k)+\sum_{j=1}^{l} B_{0 j} F_{j}(\varepsilon) Q_{j}(\varepsilon) S_{j}^{-1}(\varepsilon) \tilde{x}_{j}(k) \\
& +B_{\mathrm{R} 0} F(\varepsilon) Q(\varepsilon) S^{-1}(\varepsilon)(\tilde{x}(k-r)-\tilde{x}(k)),
\end{aligned}\right.
$$

where

$$
Q(\varepsilon)=\operatorname{blkdiag}\left\{Q_{1}(\varepsilon), Q_{2}(\varepsilon), \cdots, Q_{l}(\varepsilon), I\right\}
$$


and

$$
S(\varepsilon)=\operatorname{blkdiag}\left\{S_{1}(\varepsilon), S_{2}(\varepsilon), \cdots, S_{l}(\varepsilon), I\right\}
$$

Since all eigenvalues of $A_{i}$ are at $z=1$, it follows from Lemma 2 that

$$
\begin{aligned}
& \tilde{J}_{i}(\varepsilon)=\tilde{J}_{+1}(\varepsilon) \\
& =\left[\begin{array}{rrrr}
(1-\varepsilon) & \varepsilon & & \\
& \ddots & \ddots & \\
& & (1-\varepsilon) & \varepsilon \\
& & & (1-\varepsilon)
\end{array}\right]_{n_{i} \times n_{i}} \\
& =I_{n_{i}}+\varepsilon\left[\begin{array}{rrrr}
-1 & 1 & & \\
& \ddots & \ddots & \\
& & -1 & 1 \\
& & & -1
\end{array}\right]_{n_{i} \times n_{i}}
\end{aligned}
$$

Thus, the state equation (47) can be written in the following compact form,

(49) $\tilde{x}(k+1)=\tilde{x}(k)+\tilde{A}(\varepsilon) \tilde{x}(k)+S(\varepsilon) Q^{-1}(\varepsilon) B F(\varepsilon) Q(\varepsilon) S^{-1}(\varepsilon)(\tilde{x}(k-r)-\tilde{x}(k))$,

where $\tilde{A}(\varepsilon)$ is defined in an obvious way, and by (48) and Lemma 3, each of its elements is of the order of $\varepsilon$. Also by Lemma 3, elements of $S(\varepsilon) Q^{-1}(\varepsilon) B F(\varepsilon) Q(\varepsilon) S^{-1}(\varepsilon)$ are also of the order of $\varepsilon$.

It follows from (49) that

$$
\begin{aligned}
\tilde{x}(k-r)-\tilde{x}(k)=- & \sum_{s=0}^{r-1}\left(\tilde{A}(\varepsilon) \tilde{x}(k+s-r)+S(\varepsilon) Q^{-1}(\varepsilon) B\right. \\
& \left.\quad \times F(\varepsilon) Q(\varepsilon) S^{-1}(\varepsilon)(\tilde{x}(k+s-2 r)-\tilde{x}(k+s-r))\right) .
\end{aligned}
$$

Let us now choose a Lyapunov function

$$
V(\tilde{x})=\sum_{i=1}^{l} \kappa^{i} \tilde{x}_{i}^{\mathrm{T}} \tilde{P}_{i} \tilde{x}_{i}+\tilde{x}_{0}^{\mathrm{T}} \tilde{P}_{0} \tilde{x}_{0}:=\tilde{x}^{\mathrm{T}} \tilde{P} \tilde{x}
$$

where $\tilde{P}_{0}>0$ is such that

$$
A_{0}^{\mathrm{T}} \tilde{P}_{0} A_{0}-\tilde{P}_{0}=-I
$$

$\kappa>0$ is a constant whose value is to be determined later, and

$$
\tilde{P}=\operatorname{blkdiag}\left\{\kappa \tilde{P}_{1}, \kappa^{2} \tilde{P}_{2}, \cdots, \kappa^{l} \tilde{P}_{l}, \tilde{P}_{0}\right\}
$$

The existence of such a $\tilde{P}_{0}$ is due to the fact that $A_{0}$ is asymptotically stable. 
It follows from (50) that

$$
|\tilde{x}(k-r)-\tilde{x}(k)| \leq \varpi_{0}(\kappa) \varepsilon \sum_{s=0}^{r-1}\left(V^{\frac{1}{2}}(\tilde{x}(k+s-r))+V^{\frac{1}{2}}(\tilde{x}(k+s-2 r))\right),
$$

for some $\varpi_{0}(\kappa)>0$, dependent on $\kappa$.

The difference of $V$ along the trajectories of the closed-loop system (47) can now be evaluated as follows,

$$
\begin{aligned}
& \Delta V(\tilde{x}(k))=\sum_{i=1}^{l}\left[\kappa^{i} \tilde{x}_{i}^{\mathrm{T}}(k)\left(\tilde{J}_{i}^{\mathrm{T}}(\varepsilon) \tilde{P}_{i}(\varepsilon) \tilde{J}_{i}(\varepsilon)-\tilde{P}_{i}(\varepsilon)\right) \tilde{x}_{i}(k)\right. \\
& +2 \sum_{j=i+1}^{l} \kappa^{i} \tilde{x}_{i}^{\mathrm{T}}(k) \tilde{J}_{i}^{\mathrm{T}}(\varepsilon) \tilde{P}_{i}(\varepsilon) S_{i}(\varepsilon) Q_{i}^{-1}(\varepsilon) B_{i j} F_{j}(\varepsilon) Q_{j}(\varepsilon) S_{j}^{-1}(\varepsilon) \tilde{x}_{j}(k) \\
& +2 \kappa^{i}\left[\sum_{j=i+1}^{l} S_{i}(\varepsilon) Q_{i}^{-1}(\varepsilon) B_{i j} F_{j}(\varepsilon) Q_{j}(\varepsilon) S_{j}^{-1}(\varepsilon) \tilde{x}_{j}(k)\right]^{\mathrm{T}} \tilde{P}_{i}(\varepsilon) \\
& \times S_{i}(\varepsilon) Q_{i}^{-1}(\varepsilon) B_{\mathrm{R} i} F(\varepsilon) Q(\varepsilon) S^{-1}(\varepsilon)(\tilde{x}(k-r)-\tilde{x}(k)) \\
& +k^{i}\left[\sum_{j=i+1}^{l} S_{i}(\varepsilon) Q_{i}^{-1}(\varepsilon) B_{i j} F_{j}(\varepsilon) Q_{j}(\varepsilon) S_{j}^{-1}(\varepsilon) \tilde{x}_{j}(k)\right]^{\mathrm{T}} \tilde{P}_{i}(\varepsilon) \\
& \left.\times\left[\sum_{j=i+1}^{l} S_{i}(\varepsilon) Q_{i}^{-1}(\varepsilon) B_{i j} F_{j}(\varepsilon) Q_{j}(\varepsilon) S_{j}^{-1}(\varepsilon) \tilde{x}_{j}(k)\right]\right] \\
& -\tilde{x}_{0}^{\mathrm{T}}(k)\left(A_{0}^{\mathrm{T}} \tilde{P}_{0} A_{0}-\tilde{P}_{0}\right) \tilde{x}_{0}(k)+2 \sum_{j=1}^{n} \tilde{x}_{0}^{\mathrm{T}}(k) A_{0}^{\mathrm{T}} \tilde{P}_{0} B_{0 j} F_{j}(\varepsilon) Q_{j}(\varepsilon) S_{j}^{-1}(\varepsilon) \tilde{x}_{j}(k) \\
& +\left[\sum_{j=1}^{l} B_{0 j} F_{j}(\varepsilon) Q_{j}(\varepsilon) S_{j}^{-1}(\varepsilon) \tilde{x}_{j}(k)\right]^{\mathrm{T}} P_{0}\left[\sum_{j=1}^{l} B_{0 j} F_{j}(\varepsilon) Q_{j}(\varepsilon) S_{j}^{-1}(\varepsilon) \tilde{x}_{j}(k)\right] \\
& +2\left[\sum_{i=1}^{l} \kappa^{i} \tilde{x}_{i}^{\mathrm{T}}(k) \tilde{J}_{i}^{\mathrm{T}}(\varepsilon) \tilde{P}_{i}(\varepsilon) S_{i}(\varepsilon) Q_{i}^{-1}(\varepsilon) B_{\mathrm{R} i}+\tilde{x}_{0}^{\mathrm{T}} A_{0}^{\mathrm{T}} \tilde{P}_{0} B_{\mathrm{R} 0}\right] \\
& \times F(\varepsilon) Q(\varepsilon) S^{-1}(\varepsilon)(\tilde{x}(k-r)-\tilde{x}(k)) \\
& +(\tilde{x}(k-r)-\tilde{x}(k))^{\mathrm{T}}\left(S^{-1}(\varepsilon)\right)^{\mathrm{T}} Q^{\mathrm{T}}(\varepsilon) F^{\mathrm{T}}(\varepsilon) \\
& \times\left[\sum_{i=1}^{l} \kappa^{i} B_{\mathrm{R} i}^{\mathrm{T}}\left(Q_{i}^{-1}(\varepsilon)\right)^{\mathrm{T}} S_{i}^{\mathrm{T}}(\varepsilon) \tilde{P}_{i}(\varepsilon) S_{i}(\varepsilon) Q_{i}^{-1}(\varepsilon) B_{\mathrm{R} i}+B_{\mathrm{R} 0}^{\mathrm{T}} \tilde{P}_{0} B_{\mathrm{R} 0}\right] \\
& \times F(\varepsilon) Q(\varepsilon) S^{-1}(\varepsilon)(\tilde{x}(k-r)-\tilde{x}(k))
\end{aligned}
$$




$$
\begin{aligned}
& \leq \sum_{i=1}^{l}\left[-\varepsilon \kappa^{i} \tilde{x}_{i}^{\mathrm{T}}(k) \tilde{x}_{i}(k)\right. \\
& +2 \kappa^{i} \sum_{j=i+1}^{l} \tilde{x}_{i}^{\mathrm{T}}(k) \tilde{J}_{i}^{\mathrm{T}}(\varepsilon) \tilde{P}_{i}(\varepsilon) S_{i}(\varepsilon) Q_{i}^{-1}(\varepsilon) B_{i j} F_{j}(\varepsilon) Q_{j}(\varepsilon) S_{j}^{-1}(\varepsilon) \tilde{x}_{j}(k) \\
& +k^{i}\left[\sum_{j=i+1}^{l} S_{i}(\varepsilon) Q_{i}^{-1}(\varepsilon) B_{i j} F_{j}(\varepsilon) Q_{j}(\varepsilon) S_{j}^{-1}(\varepsilon) \tilde{x}_{j}(k)\right]^{\mathrm{T}} \tilde{P}_{i}(\varepsilon) \\
& \left.\times\left[\sum_{j=i+1}^{l} S_{i}(\varepsilon) Q_{i}^{-1}(\varepsilon) B_{i j} F_{j}(\varepsilon) Q_{j}(\varepsilon) S_{j}^{-1}(\varepsilon) \tilde{x}_{j}(k)\right]\right] \\
& -\tilde{x}_{0}^{\mathrm{T}}(k) \tilde{x}_{0}(k)+2 \sum_{j=1}^{n} \tilde{x}_{0}^{\mathrm{T}}(k) \tilde{P}_{0} B_{0 j} F_{j}(\varepsilon) Q_{j}(\varepsilon) S_{j}^{-1}(\varepsilon) \tilde{x}_{j}(k) \\
& +\left[\sum_{j=1}^{l} B_{0 j} F_{j}(\varepsilon) Q_{j}(\varepsilon) S_{j}^{-1}(\varepsilon) \tilde{x}_{j}(k)\right]^{\mathrm{T}} P_{0}\left[\sum_{j=1}^{l} B_{0 j} F_{j}(\varepsilon) Q_{j}(\varepsilon) S_{j}^{-1}(\varepsilon) \tilde{x}_{j}(k)\right] \\
& +2 \sum_{i=1}^{l}\left[\kappa^{i}\left[\sum_{j=i+1}^{l} S_{i}(\varepsilon) Q_{i}^{-1}(\varepsilon) B_{i j} F_{j}(\varepsilon) Q_{j}(\varepsilon) S_{j}^{-1}(\varepsilon) \tilde{x}_{j}(k)\right]^{\mathrm{T}} \tilde{P}_{i}(\varepsilon) S_{i}(\varepsilon) Q_{i}^{-1}(\varepsilon) B_{\mathrm{R} i}\right. \\
& \left.+k^{i} \tilde{x}_{i}^{\mathrm{T}}(k) \tilde{J}_{i}^{\mathrm{T}}(\varepsilon) \tilde{P}_{i}(\varepsilon) S_{i}(\varepsilon) Q_{i}^{-1}(\varepsilon) B_{\mathrm{R} i}+\tilde{x}_{0}^{\mathrm{T}} A_{0}^{\mathrm{T}} \tilde{P}_{0} B_{\mathrm{R} 0}\right] \\
& \times F(\varepsilon) Q(\varepsilon) S^{-1}(\varepsilon)(\tilde{x}(k-r)-\tilde{x}(k)) \\
& +(\tilde{x}(k-r)-\tilde{x}(k))^{\mathrm{T}}\left(S^{-1}(\varepsilon)\right)^{\mathrm{T}} Q^{\mathrm{T}}(\varepsilon) F^{\mathrm{T}}(\varepsilon) \\
& \times\left[\sum_{i=1}^{l} \kappa^{i} B_{\mathrm{R} i}^{\mathrm{T}}\left(Q_{i}^{-1}(\varepsilon)\right)^{\mathrm{T}} S_{i}^{\mathrm{T}}(\varepsilon) \tilde{P}_{i}(\varepsilon) S_{i}(\varepsilon) Q_{i}^{-1}(\varepsilon) B_{\mathrm{R} i}+B_{\mathrm{R} 0}^{\mathrm{T}} \tilde{P}_{0} B_{\mathrm{R} 0}\right] \\
& \times F(\varepsilon) Q(\varepsilon) S^{-1}(\varepsilon)(\tilde{x}(k-r)-\tilde{x}(k)) .
\end{aligned}
$$

By Lemma 3, the matrices defining the $\left(\tilde{x}_{i}, \tilde{x}_{j}\right)$ cross terms, besides the term $\tilde{x}_{0}^{\mathrm{T}}(k) \tilde{x}_{0}(k)$, are all of the order of $\varepsilon$. This fact, along with $(52)$, then imply that there exist a constant $\kappa>0$ and an $\varepsilon_{1}^{*} \in(0,1]$ such that,

$$
\begin{gathered}
\Delta V(\tilde{x}(k)) \leq-\frac{\varepsilon}{2} \tilde{x}^{\mathrm{T}}(k) \tilde{x}(k)+\varepsilon^{2} \varpi_{1} V^{\frac{1}{2}}(\tilde{x}(k)) \sum_{s=0}^{r-1}\left(V^{\frac{1}{2}}(\tilde{x}(k+s-r))+V^{\frac{1}{2}}(\tilde{x}(k+s-2 r))\right) \\
(54) \quad+\varepsilon^{4} \varpi_{2}\left[\sum_{s=0}^{r-1}\left(V^{\frac{1}{2}}(\tilde{x}(k+s-r))+V^{\frac{1}{2}}(\tilde{x}(k+s-2 r))\right)\right]^{2}, \quad \varepsilon \in\left(0, \varepsilon_{1}^{*}\right],
\end{gathered}
$$

for some constants $\varpi_{1}>0$ and $\varpi_{2}>0$. 
Now, let $\eta>1$ be any constant. If $V(\tilde{x}(k+s))<\eta V(\tilde{x}(k)), \forall s \in[-r, 0]$, then

$$
\begin{aligned}
\Delta V(\tilde{x}(k)) & \leq-\frac{\varepsilon}{2} \lambda_{\max }^{-1}(\tilde{P}) V(\tilde{x}(k))+\varepsilon^{2}\left(\varpi_{1} r\left(\eta+\eta^{2}\right)+\varepsilon^{2} \varpi_{2} r^{2}\left(\eta+\eta^{2}\right)^{2}\right) V(\tilde{x}(k)) \\
& =-\varepsilon\left(\frac{1}{2} \lambda_{\max }^{-1}(\tilde{P})-\varepsilon \varpi_{1} r\left(\eta+\eta^{2}\right)-\varepsilon^{3} \varpi_{2} r^{2}\left(\eta+\eta^{2}\right)^{2}\right) V(\tilde{x}(k)) .
\end{aligned}
$$

It is clear that, for any given $r \geq 0$ and $\eta>0$, there exists an $\varepsilon^{*} \in\left(0, \varepsilon_{1}^{*}\right]$, such that, for all $\varepsilon \in\left(0, \varepsilon^{*}\right]$,

$$
\Delta V(\tilde{x}(k)) \leq-\mu(\varepsilon) V(\tilde{x}(k)), \quad \text { if } V(\tilde{x}(k+s))<\eta V(\tilde{x}(k)), \quad \forall s \in[-r, 0],
$$

for some positive scalar $\mu(\varepsilon)$. It thus follows from Lemma 4 that the closed-loop system (44) is asymptotically stable.

To examine the conservativeness of the results of Theorems 1 and 2, we consider the following simple system, whose open loop system is exponentially unstable,

$$
\left\{\begin{aligned}
x(k+1) & =\beta x(k)+u(k-r), \quad \beta>1, \\
u(k) & =-\alpha x(k) .
\end{aligned}\right.
$$

We will show that, for large enough delay $r$, the system (57) is not asymptotically stable for any choice of the feedback gain $\alpha$, and thus the conditions of Theorems 1 and 2 are tight. To this end, let us consider the characteristic equation of the closed-loop system,

$$
z^{r+1}-\beta z^{r}+\alpha=0
$$

or

$$
1+\alpha \frac{1}{z^{r}(z-\beta)}=0,
$$

and examine its root loci. Plotted in Fig. 1 is the pole-zero locations of the open loop system corresponding to the characteristic equation (59). For $\alpha<0$, there will be a branch that travels on the real axis to the right of $z=\beta$. This the closed-loop system would not be stable for any values of $r$ and $\alpha \leq 0$.

We next consider the case when $\alpha>0$. In this case, two branches of the loci will travel, as the value of $\alpha$ increases, toward each other on the real axis, one starting at $z=0$ and the other at $z=\beta$. They will meet at the breakaway point

$$
z_{a}=\frac{\beta r}{r+1}
$$

which is outside the unit circle for $r$ large enough. The corresponding value of $\alpha$ at the breakaway point is

$$
\alpha_{a}=\frac{\beta^{r+1}}{r+1}\left(\frac{r}{r+1}\right)^{r} .
$$




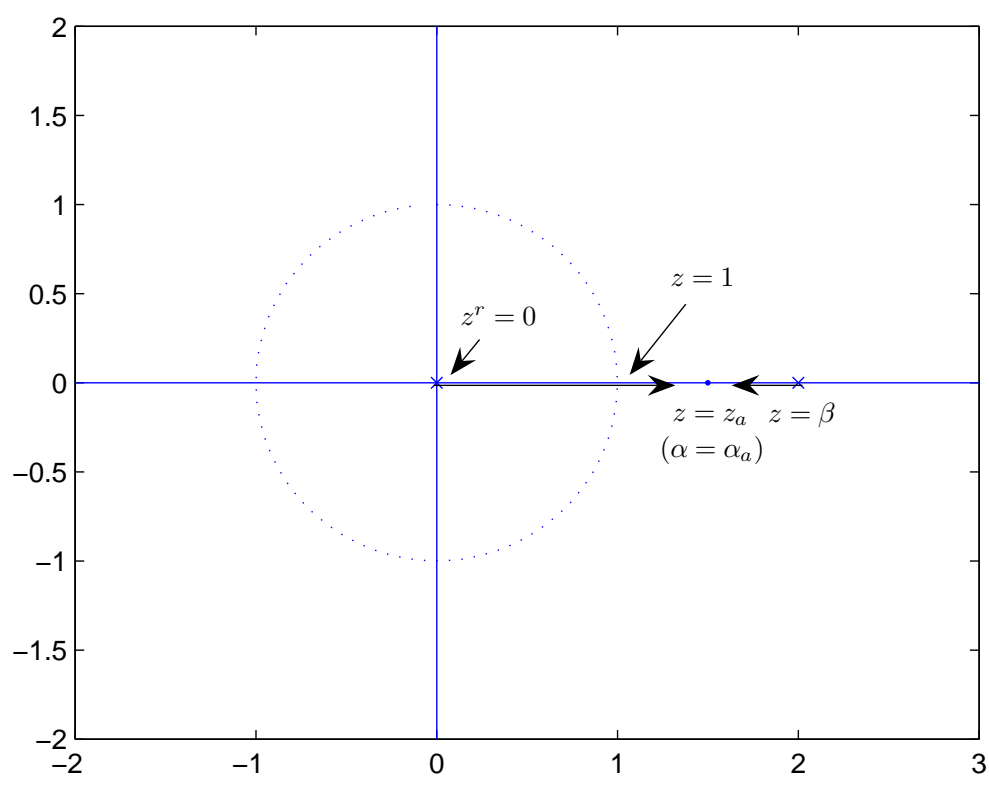

FIG. 1. Sketch of root loci of the characteristic equation (59).

Recalling that

$$
\lim _{r \rightarrow \infty}\left(\frac{r}{r+1}\right)^{r}=e^{-1}
$$

we see that the value of $\alpha_{a}$ grows unbounded as the value of $r$ increases. However, a necessary condition for the stability is $|\alpha|<1$. This implies that, for $r$ large enough, the branch of root loci that starts at $z=\beta$ will not reach $z=z_{a}$, and thus remain outside the unit circle, for $0<\alpha<1$. In conclusion, the closed-loop system (57), with a large enough delay $r$, is not asymptotically stable for any choice of the feedback gain $\alpha$.

4. Stabilization by Linear Output Feedback. For the system (1) with all eigenvalues of $A$ on or inside the unit circle, we construct the following two families of output feedback laws,

$$
\left\{\begin{aligned}
\hat{x}(k+1) & =A \hat{x}(k)+B u(k-r)-L(y(k)-C \hat{x}(k)), \\
u(k) & =F(\varepsilon) A^{r} \hat{x}(k),
\end{aligned}\right.
$$

and

$$
\left\{\begin{aligned}
\hat{x}(k+1) & =A \hat{x}(k)+B u(k-r)-L(y(k)-C \hat{x}(k)), \\
u(k) & =F(\varepsilon) \hat{x}(k),
\end{aligned}\right.
$$


where $F(\varepsilon)$ is as given by (27), and $L \in \mathbf{R}^{n \times p}$ is such that all eigenvalues of $A+L C$ are strictly inside the unit circle. We note that such a matrix $L$ exists as the pair $(A, C)$ is detectable.

The theorem below establishes that the output feedback law (60) asymptotically stabilizes the system (1) as long as all eigenvalues of $A$ are on or inside the unit circle.

THEOREM 3. Consider the closed-loop system comprising of the system (1) and the linear output feedback law (60). Let all eigenvalues of $A$ be on or inside the unit circle. Then, for any given arbitrarily large $r \geq 0$, there exists an $\varepsilon^{*}>0$, such that, for each $\varepsilon \in\left(0, \varepsilon^{*}\right]$, the closed-loop system is asymptotically stable.

Proof. Under the linear output feedback law (60), the closed-loop system is given by,

$$
\left\{\begin{array}{l}
x(k+1)=A x(k)+B F(\varepsilon) A^{r} \hat{x}(k-r), \\
\hat{x}(k+1)=A \hat{x}(k)+B F(\varepsilon) A^{r} \hat{x}(k-r)-L(y(k)-C \hat{x}(k)),
\end{array}\right.
$$

which, in the new state $(x, e)=(x, x-\hat{x})$, can be written as,

$$
\left\{\begin{array}{l}
x(k+1)=A x(k)+B F(\varepsilon) A^{r} x(k-r)-B F(\varepsilon) A^{r} e(k-r), \\
e(k+1)=(A+L C) e(k),
\end{array}\right.
$$

which in turn implies that

$A^{r} x(k-r)=x(k)-\sum_{s=0}^{r-1} A^{r-s-1} B F(\varepsilon) A^{r} x(k+s-2 r)+\sum_{s=0}^{r-1} A^{r-s-1} B F(\varepsilon) A^{r} e(k+s-2 r)$

and

$$
e(k-r)=(A+L C)^{-r} e(k) .
$$

Substitution of (64) and (65) into (63) results in

$$
\left\{\begin{aligned}
x(k+1)= & (A+B F) x(k)-B F(\varepsilon) \sum_{s=0}^{r-1} A^{r-s-1} B F(\varepsilon) A^{r} x(k+s-2 r) \\
& +B F(\varepsilon) \sum_{s=0}^{r-1} A^{r-s-1} B F(\varepsilon) A^{r} e(k+s-2 r) \\
& -B F(\varepsilon) A^{r}(A+L C)^{-r} e(k) \\
e(k+1)= & (A+L C) e(k) .
\end{aligned}\right.
$$

Without loss of generality, assume that the pair $(A, B)$ are already in the form of (22)-(23). Partitioning the state $x$ according to the structure of (22)-(23),

$$
x=\left[\begin{array}{lllll}
x_{1}^{\mathrm{T}} & x_{2}^{\mathrm{T}} & \cdots & x_{l}^{\mathrm{T}} & x_{0}^{\mathrm{T}}
\end{array}\right]^{\mathrm{T}}, \quad x_{i} \in \mathbf{R}^{n_{i}}, i \in[1, l],
$$


we rewrite the state equation (66) as follows

$$
\left\{\begin{aligned}
x_{1}(k+1)= & \left(A_{1}+B_{1} F_{1}(\varepsilon)\right) x_{1}(k)+\sum_{j=2}^{l} B_{1 j} F_{j}(\varepsilon) x_{j}(k) \\
& -B_{\mathrm{R} 1} F(\varepsilon) \sum_{s=0}^{r-1} A^{r-s-1} B F(\varepsilon) A^{r} x(k+s-2 r) \\
& +B_{\mathrm{R} 1} F(\varepsilon) \sum_{s=0}^{r-1} A^{r} B F(\varepsilon) A^{r} e(k+s-2 r) \\
& -B_{\mathrm{R} 1} F(\varepsilon) A^{r}(A+L C)^{-r} e(k) \\
x_{2}(k+1)= & \left(A_{2}+B_{2} F_{2}(\varepsilon)\right) x_{2}(k)+\sum_{j=3}^{l} B_{2 j} F_{j}(\varepsilon) x_{j}(k) \\
& -B_{\mathrm{R} 2} F(\varepsilon) \sum_{s=0}^{r-1} A^{r-s-1} B F(\varepsilon) A^{r} x(k+s-2 r) \\
& +B_{\mathrm{R} 2} F(\varepsilon) \sum_{s=0}^{r-1} A^{r-s-1} B F(\varepsilon) A^{r} e(k+s-2 r) \\
& -B_{\mathrm{R} 2} F(\varepsilon) A^{r}(A+L C)^{-r} e(k) \\
\vdots & \\
& \left(A_{l}+B_{l} F_{l}(\varepsilon)\right) x_{l}(k)-B_{\mathrm{R} l} F(\varepsilon) \sum_{s=0}^{r-1} A^{r} B F(\varepsilon) A^{r} x(k+s-2 r) \\
& +B_{\mathrm{R} l} F(\varepsilon) \sum_{s=0}^{r-1} A^{r} B F(\varepsilon) A^{r} e(k+s-2 r) \\
& -B_{\mathrm{R} l} F(\varepsilon) A^{r}(A+L C)^{-r} e(k), \\
x_{l}(k+1) & +B_{\mathrm{R} 0} F(\varepsilon) \sum_{s=0}^{r-1} A^{r} B F(\varepsilon) A^{r} e(k+s-2 r) \\
x_{0}(k+1)= & A_{0} x_{0}(k)+\sum_{j=1}^{r-1} B_{0 j} F_{j}(\varepsilon) x_{j}(k) \\
& -B_{\mathrm{R} 0} F(\varepsilon) \sum_{s=0}^{r-1} A^{r} B F(\varepsilon) A^{r} x(k+s-2 r) \\
& (A+L C) e(k) \\
& \\
& \\
& \\
& \\
&
\end{aligned}\right.
$$

where, for each $i=1$ to $l, B_{\mathrm{R} i}$ is the $i$ th row of the right hand side of (23) and $B_{\mathrm{R} 0}$ is the last row.

Now, for each $i=1$ to $l$, let $Q_{i}(\varepsilon), S_{i}(\varepsilon), \tilde{J}_{i}(\varepsilon), \tilde{P}_{i}, \gamma_{i}, \alpha_{i}, \beta_{i}$, and $\vartheta_{i}$ be the matrices $Q(\varepsilon), S(\varepsilon), \tilde{J}(\varepsilon)$ and $\tilde{P}$ and the constants $\gamma, \alpha, \beta$ and $\vartheta$ as defined in Lemmas 1-3, but for the triple $\left(A_{i}, B_{i}, F_{i}(\varepsilon)\right)$. Define a state transformation as,

$$
\tilde{x}=\left[\tilde{x}_{1}^{\mathrm{T}}, \tilde{x}_{2}^{\mathrm{T}}, \cdots, \tilde{x}_{l}^{\mathrm{T}}, \tilde{x}_{0}^{\mathrm{T}}\right]^{\mathrm{T}}, \quad \tilde{e}=e,
$$

where $\tilde{x}_{0}=x_{0}$, and, for each $i=1$ to $l, \tilde{x}_{i}=S_{i}(\varepsilon) Q_{i}^{-1}(\varepsilon) x_{i}$. 
It follows from Lemmas 1 and 2 that, under this state transformation, the state equation (67) can be written as

(69)

$$
\begin{aligned}
& \int \tilde{x}_{1}(k+1)=\tilde{J}_{1}(\varepsilon) \tilde{x}_{1}(k)+\sum_{j=2}^{l} S_{1}(\varepsilon) Q_{1}^{-1}(\varepsilon) B_{1 j} F_{j}(\varepsilon) Q_{j}(\varepsilon) S_{j}^{-1}(\varepsilon) \tilde{x}_{j}(k) \\
& -S_{1}(\varepsilon) Q_{1}^{-1}(\varepsilon) B_{\mathrm{R} 1} F(\varepsilon) \sum_{s=0}^{r-1} A^{r-s-1} B F(\varepsilon) A^{r} Q(\varepsilon) S^{-1}(\varepsilon) \tilde{x}(k+s-2 r) \\
& +S_{1}(\varepsilon) Q_{1}^{-1}(\varepsilon) B_{\mathrm{R} 1} F(\varepsilon) \sum_{s=0}^{r-1} A^{r-s-1} B F(\varepsilon) A^{r} Q(\varepsilon) S^{-1}(\varepsilon) \tilde{e}(k+s-2 r) \\
& -S_{1}(\varepsilon) Q_{1}^{-1}(\varepsilon) B_{\mathrm{R} 1} F(\varepsilon) A^{r}(A+L C)^{-r} \tilde{e}(k), \\
& \tilde{x}_{2}(k+1)=\tilde{J}_{2}(\varepsilon) \tilde{x}_{2}(k)+\sum_{j=3}^{l} S_{2}(\varepsilon) Q_{2}(\varepsilon) B_{2 j} F_{j}(\varepsilon) Q_{j}(\varepsilon) S_{j}^{-1}(\varepsilon) \tilde{x}_{j}(k) \\
& -S_{2}(\varepsilon) Q_{2}^{-1}(\varepsilon) B_{\mathrm{R} 2} F(\varepsilon) \sum_{s=0}^{r-1} A^{r-s-1} B F(\varepsilon) A^{r} Q(\varepsilon) S^{-1}(\varepsilon) \tilde{x}(k+s-2 r) \\
& -S_{2}(\varepsilon) Q_{2}^{-1}(\varepsilon) B_{\mathrm{R} 2} F(\varepsilon) e^{A \tau} e^{-(A+L C) \tau} \tilde{e}(k), \\
& +S_{2}(\varepsilon) Q_{2}^{-1}(\varepsilon) B_{\mathrm{R} 2} F(\varepsilon) \sum_{s=0}^{r-1} A^{r-s-1} B F(\varepsilon) A^{r} Q(\varepsilon) S^{-1}(\varepsilon) \tilde{e}(k+s-2 r) \\
& -S_{2}(\varepsilon) Q_{2}^{-1}(\varepsilon) B_{\mathrm{R} 2} F(\varepsilon) A^{r}(A+L C)^{-r} \tilde{e}(k), \\
& \vdots \\
& \tilde{x}_{l}(k+1)=\tilde{J}_{l}(\varepsilon) \tilde{x}_{l}(k) \\
& -S_{l}(\varepsilon) Q_{l}^{-1}(\varepsilon) B_{\mathrm{R} l} F(\varepsilon) \sum_{s=0}^{r-1} A^{r-s-1} B F(\varepsilon) A^{r} Q(\varepsilon) S^{-1}(\varepsilon) \tilde{x}(k+s-2 r) \\
& +S_{l}(\varepsilon) Q_{l}^{-1}(\varepsilon) B_{\mathrm{R} l} F(\varepsilon) \sum_{s=0}^{r-1} A^{r-s-1} B F(\varepsilon) A^{r} Q(\varepsilon) S^{-1}(\varepsilon) \tilde{e}(k+s-2 r) \\
& -S_{l}(\varepsilon) Q_{l}^{-1}(\varepsilon) B_{\mathrm{R} 2} F(\varepsilon) A^{r}(A+L C)^{-r} \tilde{e}(k), \\
& \tilde{x}_{0}(k+1)=A_{0} \tilde{x}_{0}(k)+\sum_{j=1}^{l} B_{0 j} F_{j}(\varepsilon) Q_{j}(\varepsilon) S_{j}^{-1}(\varepsilon) \tilde{x}_{j}(k) \\
& -B_{\mathrm{R} 0} F(\varepsilon) \sum_{s=0}^{r-1} A^{r-s-1} B F(\varepsilon) A^{r} Q(\varepsilon) S^{-1}(\varepsilon) \tilde{x}(k+s-2 r), \\
& +B_{\mathrm{R} 0} F(\varepsilon) \sum_{s=0}^{r-1} A^{r-s-1} B F(\varepsilon) A^{r} Q(\varepsilon) S^{-1}(\varepsilon) \tilde{e}(k+s-2 r) \\
& -B_{\mathrm{R} 0} F(\varepsilon) A^{r}(A+L C)^{-r} \tilde{e}(k), \\
& \tilde{e}(k+1)=(A+L C) \tilde{e}(k),
\end{aligned}
$$

where

$$
Q(\varepsilon)=\operatorname{blkdiag}\left\{Q_{1}(\varepsilon), Q_{2}(\varepsilon), \cdots, Q_{l}(\varepsilon), I\right\}
$$

and

$$
S(\varepsilon)=\operatorname{blkdiag}\left\{S_{1}(\varepsilon), S_{2}(\varepsilon), \cdots, S_{l}(\varepsilon), I\right\} .
$$


Let us choose the Lyapunov function

$$
V(\tilde{x}, \tilde{e})=\sum_{i=1}^{l} \kappa^{i} \tilde{x}_{i}^{\mathrm{T}} \tilde{P}_{i} \tilde{x}_{i}+\tilde{x}_{0}^{\mathrm{T}} \tilde{P}_{0} \tilde{x}_{0}+\kappa^{l+1} \tilde{e}^{\mathrm{T}} \tilde{Q} \tilde{e}:=\tilde{x}^{\mathrm{T}} \tilde{P} \tilde{x}+\kappa^{l+1} \tilde{e}^{\mathrm{T}} \tilde{Q} \tilde{e}
$$

where $\tilde{P}_{0}>0$ and $\tilde{Q}>0$ are the solutions to the Lyapunov equations

$$
A_{0}^{\mathrm{T}} \tilde{P}_{0} A_{0}-\tilde{P}_{0}=-I
$$

and

$$
(A+L C)^{\mathrm{T}} \tilde{Q}(A+L C)-\tilde{Q}=-I
$$

respectively, $\kappa>0$ is a constant whose value is to be determined later, and

$$
\tilde{P}=\operatorname{blkdiag}\left\{\kappa \tilde{P}_{1}, \kappa^{2} \tilde{P}_{2}, \cdots, \kappa^{l} \tilde{P}_{l}, \tilde{P}_{0}\right\} .
$$

The existence of such $\tilde{P}_{0}$ and $\tilde{Q}$ is due to the fact that both $A_{0}$ and $A+L C$ are asymptotically stable.

The difference of $V$ along the trajectories of the closed-loop system (69) can be evaluated as follows

$$
\begin{aligned}
& \Delta V(\tilde{x}(k))=\sum_{i=1}^{l}\left[\kappa^{i} \tilde{x}_{i}^{\mathrm{T}}(k)\left(\tilde{J}_{i}^{\mathrm{T}}(\varepsilon) \tilde{P}_{i}(\varepsilon) \tilde{J}_{i}(\varepsilon)-\tilde{P}_{i}(\varepsilon)\right) \tilde{x}_{i}(k)\right. \\
&+2 \sum_{j=i+1}^{l} \kappa^{i} \tilde{x}_{i}^{\mathrm{T}}(k) \tilde{J}_{i}^{\mathrm{T}}(\varepsilon) \tilde{P}_{i}(\varepsilon) S_{i}(\varepsilon) Q_{i}^{-1}(\varepsilon) B_{i j} F_{j}(\varepsilon) Q_{j}(\varepsilon) S_{j}^{-1}(\varepsilon) \tilde{x}_{j}(k) \\
&-2 \kappa^{i} \tilde{x}_{i}^{\mathrm{T}}(k) \tilde{J}_{i}(\varepsilon) \tilde{P}_{i}(\varepsilon) S_{i}(\varepsilon) Q_{i}^{-1}(\varepsilon) B_{\mathrm{R} i} F(\varepsilon) A^{r}(A+L C)^{-1} \tilde{e}(k) \\
&-2 \kappa^{i}\left[\sum_{j=i+1}^{l} S_{i}(\varepsilon) Q_{i}^{-1}(\varepsilon) B_{i j} F_{j}(\varepsilon) Q_{j}(\varepsilon) S_{j}^{-1}(\varepsilon) \tilde{x}_{j}(k)\right]^{\mathrm{T}} \tilde{P}_{i}(\varepsilon) \\
& \quad \times S_{i}(s) Q_{i}^{-1}(\varepsilon) B_{\mathrm{R} i} F(\varepsilon) A^{r}(A+L C)^{-r} \tilde{e}(k) \\
&-2 \kappa^{i}\left[\sum_{j=i+1}^{l} S_{i}(\varepsilon) Q_{i}^{-1}(\varepsilon) B_{i j} F_{j}(\varepsilon) Q_{j}(\varepsilon) S_{j}^{-1}(\varepsilon) \tilde{x}_{j}(k)\right. \\
& \quad \times S_{i}(\varepsilon) Q_{i}^{-1}(\varepsilon) B_{\mathrm{R} i} F(\varepsilon) \sum_{s=0}^{r-1} A^{r-s-1} B F(\varepsilon) A^{r} Q(\varepsilon) S^{-1}(\varepsilon) \tilde{x}(k+s-2 r) \\
&-2 \kappa^{i}\left[\sum_{j=i+1}^{l} S_{i}(\varepsilon) Q_{i}^{-1}(\varepsilon) B_{i j} F_{j}(\varepsilon) Q_{j}(\varepsilon) S_{j}^{-1}(\varepsilon) \tilde{x}_{j}(k)\right]^{\mathrm{T}} \tilde{P}_{i}(\varepsilon) \\
& \times S_{i}(\varepsilon) Q_{i}^{-1}(\varepsilon) B_{\mathrm{R} i} F(\varepsilon) \sum_{s=0}^{r-1} A^{r-s-1} B F(\varepsilon) A^{r} Q(\varepsilon) S^{-1}(\varepsilon) \tilde{e}(k+s-2 r)
\end{aligned}
$$




$$
\begin{aligned}
& +2 \kappa^{i} \tilde{e}^{\mathrm{T}}(k)\left(S_{i}(\varepsilon) Q_{i}^{-1}(\varepsilon) B_{\mathrm{R} i} F(\varepsilon) A^{r}(A+L C)^{-r}\right)^{\mathrm{T}} \tilde{P}_{i}(\varepsilon) S_{i}(\varepsilon) Q_{i}^{-1}(\varepsilon) B_{\mathrm{R} i} \\
& \times F(\varepsilon) \sum_{s=0}^{r-1} A^{r-s-1} B F(\varepsilon) A^{r} Q(\varepsilon) S^{-1}(\varepsilon) \tilde{x}(k+s-2 r) \\
& -2 \kappa^{i} \tilde{e}^{\mathrm{T}}(k)\left(S_{i}(\varepsilon) Q_{i}^{-1}(\varepsilon) B_{\mathrm{R} i} F(\varepsilon) A^{r}(A+L C)^{-r}\right)^{\mathrm{T}} \tilde{P}_{i}(\varepsilon) S_{i}(\varepsilon) Q_{i}^{-1}(\varepsilon) B_{\mathrm{R} i} \\
& \times F(\varepsilon) \sum_{s=0}^{r-1} A^{r-s-1} B F(\varepsilon) A^{r} Q(\varepsilon) S^{-1}(\varepsilon) \tilde{e}(k+s-2 r) \\
& +k^{i}\left[\sum_{j=i+1}^{l} S_{i}(\varepsilon) Q_{i}^{-1}(\varepsilon) B_{i j} F_{j}(\varepsilon) Q_{j}(\varepsilon) S_{j}^{-1}(\varepsilon) \tilde{x}_{j}(k)\right]^{\mathrm{T}} \tilde{P}_{i}(\varepsilon) \\
& \times\left[\sum_{j=i+1}^{l} S_{i}(\varepsilon) Q_{i}^{-1}(\varepsilon) B_{i j} F_{j}(\varepsilon) Q_{j}(\varepsilon) S_{j}^{-1}(\varepsilon) \tilde{x}_{j}(k)\right] \\
& +\kappa^{i}\left(S_{i}(s) Q_{i}^{-1}(\varepsilon) B_{\mathrm{R} i} F(\varepsilon) A^{r}(A+L C)^{-r} \tilde{e}(k)\right)^{\mathrm{T}} \tilde{P}_{i}(\varepsilon) \\
& \left.\times S_{i}(s) Q_{i}^{-1}(\varepsilon) B_{\mathrm{R} i} F(\varepsilon) A^{r}(A+L C)^{-r} \tilde{e}(k)\right] \\
& -\tilde{x}_{0}^{\mathrm{T}}(k)\left(A_{0}^{\mathrm{T}} \tilde{P}_{0} A_{0}-\tilde{P}_{0}\right) \tilde{x}_{0}(k)+2 \sum_{j=1}^{l} \tilde{x}_{0}^{\mathrm{T}}(k) A_{0}^{\mathrm{T}} \tilde{P}_{0} B_{0 j} F_{j}(\varepsilon) Q_{j}(\varepsilon) S_{j}^{-1}(\varepsilon) \tilde{x}_{j}(k) \\
& -2 \tilde{x}_{0}^{\mathrm{T}}(k) A_{0}^{\mathrm{T}} \tilde{P}_{0} B_{\mathrm{R} 0} F(\varepsilon) A^{r}(A+L C)^{-r} \tilde{e}(k) \\
& -2\left[\sum_{j=1}^{l} B_{0 j} F_{j}(\varepsilon) Q_{j}(\varepsilon) S_{j}^{-1}(\varepsilon) \tilde{x}_{j}(k)\right]^{\mathrm{T}} \tilde{P}_{0} B_{\mathrm{R} 0} F(\varepsilon) A^{r}(A+L C)^{-r} \tilde{e}(k) \\
& +\left[\sum_{j=1}^{l} B_{0 j} F_{j}(\varepsilon) Q_{j}(\varepsilon) S_{j}^{-1}(\varepsilon) \tilde{x}_{j}(k)\right]^{\mathrm{T}} \tilde{P}_{0}\left[\sum_{j=1}^{l} B_{0 j} F_{j}(\varepsilon) Q_{j}(\varepsilon) S_{j}^{-1}(\varepsilon) \tilde{x}_{j}(k)\right] \\
& +\left(B_{\mathrm{R} 0} F(\varepsilon) A^{r}(A+L C)^{-r} \tilde{e}(k)\right)^{\mathrm{T}} \tilde{P}_{0} B_{\mathrm{R} 0} F(\varepsilon) A^{r}(A+L C)^{-r} \tilde{e}(k) \\
& -2\left[\sum_{i=1}^{l} \kappa^{i} \tilde{x}_{i}^{\mathrm{T}}(k) \tilde{J}_{i}^{\mathrm{T}}(\varepsilon) \tilde{P}_{i}(\varepsilon) S_{i}(\varepsilon) Q_{i}^{-1}(\varepsilon) B_{\mathrm{R} i}+\tilde{x}_{0}^{\mathrm{T}} A_{0}^{\mathrm{T}} \tilde{P}_{0} B_{\mathrm{R} 0}\right] \\
& \times F(\varepsilon) \sum_{s=0}^{r-1} A^{r-s-1} B F(\varepsilon) A^{r} Q(\varepsilon) S^{-1}(\varepsilon) \tilde{x}(k+s-2 r) \\
& +2\left[\sum_{i=1}^{l} \kappa^{i} \tilde{x}_{i}^{\mathrm{T}}(k) \tilde{J}_{i}^{\mathrm{T}}(\varepsilon) \tilde{P}_{i}(\varepsilon) S_{i}(\varepsilon) Q_{i}^{-1}(\varepsilon) B_{\mathrm{R} i}+\tilde{x}_{0}^{\mathrm{T}} A_{0}^{\mathrm{T}} \tilde{P}_{0} B_{\mathrm{R} 0}\right] \\
& \times F(\varepsilon) \sum_{s=0}^{r-1} A^{r-s-1} B F(\varepsilon) A^{r} Q(\varepsilon) S^{-1}(\varepsilon) \tilde{e}(k+s-2 r) \\
& +\left[F(\varepsilon) \sum_{s=0}^{r-1} A^{r-s-1} B F(\varepsilon) A^{r} Q(\varepsilon) S^{-1}(\varepsilon) \tilde{x}(k+s-2 r)\right]^{\mathrm{T}} \\
& \times\left[\sum_{i=1}^{l} \kappa^{i} B_{\mathrm{R} i}^{\mathrm{T}}\left(Q_{i}^{-1}(\varepsilon)\right)^{\mathrm{T}} S_{i}^{\mathrm{T}}(\varepsilon) \tilde{P}_{i}(\varepsilon) S_{i}(\varepsilon) Q_{i}^{-1}(\varepsilon) B_{\mathrm{R} i}+B_{\mathrm{R} 0}^{\mathrm{T}} \tilde{P}_{0} B_{\mathrm{R} 0}\right]
\end{aligned}
$$




$$
\begin{aligned}
& \times\left[F(\varepsilon) \sum_{s=0}^{r-1} A^{r-s-1} B F(\varepsilon) A^{r} Q(\varepsilon) S^{-1}(\varepsilon) \tilde{x}(k+s-2 r)\right] \\
+ & {\left[F(\varepsilon) \sum_{s=0}^{r-1} A^{r-s-1} B F(\varepsilon) A^{r} Q(\varepsilon) S^{-1}(\varepsilon) \tilde{e}(k+s-2 r)\right]^{\mathrm{T}} } \\
\times & {\left[\sum_{i=1}^{l} \kappa^{i} B_{\mathrm{R} i}^{\mathrm{T}}\left(Q_{i}^{-1}(\varepsilon)\right)^{\mathrm{T}} S_{i}^{\mathrm{T}}(\varepsilon) \tilde{P}_{i}(\varepsilon) S_{i}(\varepsilon) Q_{i}^{-1}(\varepsilon) B_{\mathrm{R} i}+B_{\mathrm{R} 0}^{\mathrm{T}} \tilde{P}_{0} B_{\mathrm{R} 0}\right] } \\
\times & {\left[F(\varepsilon) \sum_{s=0}^{r-1} A^{r-s-1} B F(\varepsilon) A^{r} Q(\varepsilon) S^{-1}(\varepsilon) \tilde{e}(k+s-2 r)\right] } \\
-2 & {\left[F(\varepsilon) \sum_{s=0}^{r-1} A^{r-s-1} B F(\varepsilon) A^{r} Q(\varepsilon) S^{-1}(\varepsilon) \tilde{x}(k+s-2 r)\right] } \\
\times & {\left[\sum_{i=1}^{l} \kappa^{i} B_{\mathrm{R} i}^{\mathrm{T}}\left(Q_{i}^{-1}(\varepsilon)\right)^{\mathrm{T}} S_{i}^{\mathrm{T}}(\varepsilon) \tilde{P}_{i}(\varepsilon) S_{i}(\varepsilon) Q_{i}^{-1}(\varepsilon) B_{\mathrm{R} i}+B_{\mathrm{R} 0}^{\mathrm{T}} \tilde{P}_{0} B_{\mathrm{R} 0}\right] } \\
\times & {\left[F(\varepsilon) \sum_{s=0}^{r-1} A^{r-s-1} B F(\varepsilon) A^{r} Q(\varepsilon) S^{-1}(\varepsilon) \tilde{e}(k+s-2 r)\right] } \\
+\kappa & {\left[\tilde{e}^{\mathrm{T}}(k)\left((A+L C)^{\mathrm{T}} \tilde{Q}(A+L C)-\tilde{Q}\right) \tilde{e}(k)\right.}
\end{aligned}
$$

In view of Lemmas 3 , the matrices defining the $\left(\tilde{x}_{i}, \tilde{x}_{j}\right)$ and $\left(\tilde{x}_{i}, \tilde{e}\right)$ cross terms, other than the terms $\tilde{x}_{0}^{\mathrm{T}}(k)\left(A_{0}^{\mathrm{T}} \tilde{P}_{0} A_{0}-\tilde{P}_{0}\right) \tilde{x}_{0}(k)$ and $\tilde{e}^{\mathrm{T}}(k)\left((A+L C)^{\mathrm{T}} \tilde{Q}(A+L C)\right.$ $-\tilde{Q}) \tilde{e}(k)$, are all in the order of $\varepsilon$. It is then straightforward to verify that, there exists a constant $\kappa>0$ and an $\varepsilon_{1}^{*} \in(0,1]$ such that,

$$
\begin{aligned}
\Delta V(\tilde{x}(k), \tilde{e}(k)) \leq & -\frac{\varepsilon}{2} \tilde{x}^{\mathrm{T}}(k) \tilde{x}(k)-\frac{1}{2} \tilde{e}^{\mathrm{T}}(k) \tilde{e}(k) \\
& +\tilde{x}^{\mathrm{T}}(k) M_{1}(\varepsilon) F(\varepsilon) \sum_{s=0}^{r-1} A^{r-s-1} B F(\varepsilon) A^{r} Q(\varepsilon) S^{-1}(\varepsilon) \tilde{x}(k+s-2 r) \\
& +\tilde{x}^{\mathrm{T}}(k) M_{2}(\varepsilon) F(\varepsilon) \sum_{s=0}^{r-1} A^{r-s-1} B F(\varepsilon) A^{r} Q(\varepsilon) S^{-1}(\varepsilon) \tilde{e}(k+s-2 r) \\
+ & \tilde{e}^{\mathrm{T}}(k) M_{3}(\varepsilon) F(\varepsilon) \sum_{s=0}^{r-1} A^{r-s-1} B F(\varepsilon) A^{r} Q(\varepsilon) S^{-1}(\varepsilon) \tilde{x}(k+s-2 r) \\
+ & \tilde{e}^{\mathrm{T}}(k) M_{4}(\varepsilon) F(\varepsilon) \sum_{s=0}^{r-1} A^{r-s-1} B F(\varepsilon) A^{r} Q(\varepsilon) S^{-1}(\varepsilon) \tilde{e}(k+s-2 r) \\
+ & {\left[\sum_{s=0}^{r-1} A^{r-s-1} B F(\varepsilon) A^{r} Q(\varepsilon) S^{-1}(\varepsilon) \tilde{x}(k+s-2 r)\right]^{\mathrm{T}}(\varepsilon) M_{5}(\varepsilon) } \\
& \times F^{\mathrm{T}}(\varepsilon) \sum_{s=0}^{r-1} A^{r-s-1} B F(\varepsilon) A^{r} Q(\varepsilon) S^{-1}(\varepsilon) \tilde{x}(k+s-2 r)
\end{aligned}
$$




$$
\begin{aligned}
& +\left[\sum_{s=0}^{r-1} A^{r-s-1} B F(\varepsilon) A^{r} Q(\varepsilon) S^{-1}(\varepsilon) \tilde{e}(k+s-2 r)\right]^{\mathrm{T}} F^{\mathrm{T}}(\varepsilon) M_{5}(\varepsilon) \\
& \quad \times F(\varepsilon) \sum_{s=0}^{r-1} A^{r-s-1} B F(\varepsilon) A^{r} Q(\varepsilon) S^{-1}(\varepsilon) \tilde{e}(k+s-2 r) \\
& +\left[\sum_{s=0}^{r-1} A^{r-s-1} B F(\varepsilon) A^{r} Q(\varepsilon) S^{-1}(\varepsilon) \tilde{x}(k+s-2 r)\right]^{\mathrm{T}} F^{\mathrm{T}}(\varepsilon) M_{6}(\varepsilon) \\
& \quad \times F(\varepsilon) \sum_{s=0}^{r-1} A^{r-s-1} B F(\varepsilon) A^{r} Q(\varepsilon) S^{-1}(\varepsilon) \tilde{e}(k+s-2 r),
\end{aligned}
$$

where matrices $M_{i}(\varepsilon), i=1,2, \cdots, 6$, are defined in an obvious way and are all of order $\varepsilon^{0}$.

By using Lemma 3 again and (40), we can easily see that,

$$
\begin{aligned}
\Delta V(\tilde{x}(k), \tilde{e}(k)) \leq & -\frac{\varepsilon}{2} \min \left\{\lambda_{\max }^{-1}(\tilde{P}), \lambda_{\max }^{-1}\left(\kappa^{l+1} \tilde{Q}\right)\right\} V(\tilde{x}(k), \tilde{e}(k)) \\
& +\varepsilon^{2} \varpi_{1}(r) V^{\frac{1}{2}}(\tilde{x}(k), \tilde{e}(k)) \sum_{s=0}^{r-1}|A|^{r-s-1} V^{\frac{1}{2}}(\tilde{x}(k+s-2 r), \tilde{e}(k+s-2 r)) \\
(73) \quad & +\varepsilon^{4} \varpi_{2}(r)\left[\sum_{s=0}^{r-1}|A|^{r-s-1} V^{\frac{1}{2}}(\tilde{x}(k+s-2 r), \tilde{e}(k+s-2 r))\right]^{2}, \quad \varepsilon \in\left(0, \varepsilon_{1}^{*}\right],
\end{aligned}
$$

for some $\varpi_{1}(r), \varpi_{2}(r)>0$, both independent of $\varepsilon$.

Now, let $\eta>1$ be any constant. If $V(\tilde{x}(k+s), \tilde{e}(k+s)<\eta V(\tilde{x}(k), \tilde{e}(k)), \forall s \in$ $[-r, 0]$

$$
\begin{aligned}
\Delta V(\tilde{x}(k), \tilde{e}(k)) \leq & -\frac{\varepsilon}{2} \min \left\{\lambda_{\max }^{-1}(\tilde{P}), \lambda_{\max }^{-1}\left(\kappa^{l+1} \tilde{Q}\right)\right\} V(\tilde{x}(k), \tilde{e}(k)) \\
& +\varepsilon^{2} \varpi_{1}(r) \eta\left[\sum_{s=0}^{r-1}|A|^{r-s-1}\right] V(\tilde{x}(k), \tilde{e}(k)) \\
& +\varepsilon^{4} \varpi_{2}(r) \eta^{2}\left[\sum_{s=0}^{r-1}|A|^{r-s-1}\right]^{2} V(\tilde{x}(k), \tilde{e}(k)), \quad \varepsilon \in\left(0, \varepsilon_{1}^{*}\right] .
\end{aligned}
$$

It is now clear that, for any given $r$, there exists an $\varepsilon^{*} \in(0,1]$ such that, for all $\varepsilon \in\left(0, \varepsilon^{*}\right]$,

$$
\Delta V(\tilde{x}(k), \tilde{e}(k)) \leq-\sqrt{\varepsilon} V(\tilde{x}(k), \tilde{e}(k)) .
$$

It then follows from Lemma 4 that the closed-loop system (62) is asymptotically stable. This completes the proof.

Theorem 3 involves the linear output feedback law (60), which depends explicitly on the value of $r$. The theorem below shows that if all eigenvalues of $A$ are at $z=1$ or strictly inside the unit circle, then the system (1) can be asymptotically stabilized 
by a linear output feedback law (61), which does not require the explicit knowledge of $\tau$.

TheOREm 4. Consider the closed-loop system comprising of the system (1) and the linear output feedback law (61). Let all eigenvalues of $A$ be at $z=1$ or strictly inside the unit circle. Then, for any given arbitrarily large $r \geq 0$, there exists an $\varepsilon^{*}>0$, such that, for each $\varepsilon \in\left(0, \varepsilon^{*}\right]$, the closed-loop system is asymptotically stable.

Proof. The proof follows the procedure of that of Theorem 3. But, instead of using (64) to create the stabilizing feedback action $F(\varepsilon) x(k)$ from the delayed feedback $F A^{r} \hat{x}(k-r)$, it uses a formula similar to (50) in the proof of Theorem 2 to create the stabilizing control action $F(\varepsilon) x(k)$ directly from the delayed feedback $F(\varepsilon) \hat{x}(k-r)$. As in the proof of Theorem 2, this is possible because, under the new state $(\tilde{x}, \tilde{e})$, the matrices $\tilde{J}_{i}(\varepsilon)-I_{n_{i}}$ 's, where $\tilde{J}_{i}(\varepsilon)$ is the matrix $\tilde{J}(\varepsilon)$ 's for the triples $\left(A_{i}, B_{i}, F_{i}(\varepsilon)\right)$ as defined in Lemma 2, are of the order of $\varepsilon$.

5. Semi-Global Stabilization by Saturated Linear Feedback. We next consider the system (1) subject to actuator saturation,

$$
\left\{\begin{aligned}
x(k+1) & =A x(k)+B \operatorname{sat}(u(k-r)), \quad x(s)=\phi(s), s \in[-r, 0], \\
y(k) & =C x(k),
\end{aligned}\right.
$$

where sat : $\mathbf{R}^{m} \rightarrow \mathbf{R}^{m}$ is the standard vector valued saturation function, i.e.,

$$
\operatorname{sat}(u)=\left[\begin{array}{llll}
\operatorname{sat}\left(u_{1}\right) & \operatorname{sat}\left(u_{2}\right) & \cdots & \operatorname{sat}\left(u_{m}\right)
\end{array}\right]^{\mathrm{T}}, \quad \operatorname{sat}\left(u_{i}\right)=\operatorname{sgn}\left(u_{i}\right) \min \left\{u_{i}, 1\right\} .
$$

Here, we have slightly abused the notation by using "sat" to denote both the scalar valued and vector valued saturation functions. We also note that it is without loss of generality to assume that the saturation level is unity. Non-unity saturation level can be absorbed into the matrix $B$ and the control $u$.

Our next theorem establishes semi-global stabilizability of the system (76) by linear state or output feedback.

TheOREM 5. Consider the system (76) for a given arbitrarily large $r$.

a) If all eigenvalues of $A$ are on or strictly inside the unit circle, then, either the family of linear state feedback laws (25) or the family of linear output feedback laws (60) semi-globally stabilizes the system at the origin, i.e., for any a priori given bounded set $\Phi \subset \mathcal{C}_{n, r}$ (for state feedback) or $\Phi \subset \mathcal{C}_{2 n, r}$ (for output feedback), there exists an $\varepsilon^{*}>0$, such that, for each $\varepsilon \in\left(0, \varepsilon^{*}\right]$, the closed-loop system is asymptotically stable at the origin with $\Phi$ contained in the domain of attraction.

b) If eigenvalues of $A$ are either at $z=1$ or strictly inside the unit circle, then, either the family of linear state feedback laws (26) or the family of linear output feedback laws (61) semi-globally stabilizes the system at the origin. 
Proof. We first prove Item a) of the theorem. Under the linear state feedback law (25), the closed-loop system can be written as

$$
x(k+1)=A x(k)+B \operatorname{sat}\left(F(\varepsilon) A^{r} x(k-r)\right) .
$$

As in the proof of Theorem 1, we consider a state transformation $\tilde{x}=S(\varepsilon) Q^{-1}(\varepsilon) x$ and Lyapunov function $V(\tilde{x})=\tilde{x}^{\mathrm{T}} \tilde{P} \tilde{x}$. Let

$$
\rho^{*} \geq \sup _{x \in \Phi, s \in[-r, 0], \varepsilon \in(0,1]} \tilde{x}^{\mathrm{T}}(s) \tilde{P} \tilde{x}(s) .
$$

Such a $\rho^{*}$ exists since $\Phi$ is bounded and $\left|Q^{-1}(\varepsilon)\right| \leq 1+\sum_{i=1}^{l} \vartheta_{i}$ by Lemma 3. Clearly, $x \in \Psi$ implies that

$$
\tilde{x} \in M_{V}\left(\rho^{*}\right):=\left\{\phi \in \mathcal{C}_{n, r}: V(\phi(s)) \leq \rho, \forall s \in[-r, 0]\right\} .
$$

Let $\varepsilon_{1}^{*} \in(0,1]$ be such that

$$
M_{V}\left(\rho^{*}\right) \subset \mathcal{L}\left(F(\varepsilon) A^{r}\right):=\left\{\tilde{x} \in \mathcal{C}_{n, r}:\left|F(\varepsilon) A^{r} Q(\varepsilon) S^{-1}(\varepsilon) \tilde{x}\right|_{\infty} \leq 1\right\},
$$

where $|\cdot|_{\infty}$ denotes the infinity norm of a vector. The existence of such an $\varepsilon_{1}^{*}$ is due to (40) and the fact that $M_{V}\left(\rho^{*}\right)$ is independent of $\varepsilon$.

It is clear that, within $M_{V}\left(\rho^{*}\right)$, the closed-loop system (77) reduces to a linear system, which in the transformed state $\tilde{x}$ is given as (33). Thus, as established in the proof of Theorem 1 , for any given $\eta>1$, there exist an $\eta>1$ and an $\varepsilon^{*} \in\left(0, \varepsilon_{1}^{*}\right]$ such that, within $M_{V}\left(\rho^{*}\right)$,

$$
V(\tilde{x}(k)) \leq-\mu(\varepsilon) V(\tilde{x}(k)), \quad \text { if } V(\tilde{x}(k+s))<\eta V(\tilde{x}(k)), \forall s \in[-r, 0],
$$

for some positive scalar $\mu(\varepsilon)$. It then follows from Lemma 4 that the closed-loop system (77) is asymptotically stable at $x \equiv 0$ with $M_{V}\left(\rho^{*}\right) \supset \Phi$ contained in the domain of attraction.

The output feedback result can be established in a similar way. Under the linear output feedback law $(60)$, the closed-loop system can be written in the state $(x, e)=$ $(x, x-\hat{e})$ as

$$
\left\{\begin{aligned}
x(k+1) & =A x(k)+B \operatorname{sat}\left(F(\varepsilon) A^{r} x(k-r)-F(\varepsilon) A^{r} e(k-r)\right), \\
e(k) & =(A+L C) e(k),
\end{aligned}\right.
$$

As in the proof of Theorem 3, we will work with the transformed state $(\tilde{x}, \tilde{e})$ and define the Lyapunov function as

$$
V(\tilde{x}, \tilde{e})=\tilde{x}^{\mathrm{T}} \tilde{P} \tilde{x}+\kappa^{l+1} \tilde{e} \tilde{Q} \tilde{e} .
$$

Let

$$
\rho^{*} \geq \sup _{(x, \hat{x}) \in \Phi, s \in[-r, 0], \varepsilon \in(0,1]}\left\{\tilde{x}^{\mathrm{T}}(s) \tilde{P} \tilde{x}(s)+\kappa^{l+1} \tilde{e}^{\mathrm{T}}(s) \tilde{Q} \tilde{e}(s)\right\}
$$


Such a $\rho^{*}$ exists since $\Phi$ is bounded and $\left|Q^{-1}(\varepsilon)\right| \leq 1+\sum_{i=1}^{l} \vartheta_{i}$ by Lemma 3. Clearly, $(x, \hat{x}) \in \Phi$ implies that

$$
(\tilde{x}, \tilde{e}) \in M_{V}\left(\rho^{*}\right):=\left\{\phi \in \mathcal{C}_{2 n, r}: V(\phi(s)) \leq \rho, \forall s \in[-r, 0]\right\} .
$$

Let $\varepsilon_{1}^{*} \in(0,1]$ be such that

$$
\begin{aligned}
M_{V}\left(\rho^{*}\right) & \subset \mathcal{L}\left(\left[F(\varepsilon) A^{r}-F(\varepsilon) A^{r}\right]\right) \\
& :=\left\{(\tilde{x}, \tilde{e}) \in \mathcal{C}_{2 n, r}:\left|F(\varepsilon) A^{r} Q(\varepsilon) S^{-1}(\varepsilon) \tilde{x}-F(\varepsilon) A^{r} \tilde{e}\right|_{\infty} \leq 1\right\} .
\end{aligned}
$$

The existence of such an $\varepsilon_{1}^{*}$ is due to (40) and the fact that $M_{V}\left(\rho^{*}\right)$ is independent of $\varepsilon$.

It is clear that, within $M_{V}\left(\rho^{*}\right)$, the closed-loop system (80) reduces to a linear system, which in the transformed state $(\tilde{x}, \tilde{e})$ is given as $(69)$. Thus, as shown in the proof of Theorem 3 , there exist, for any given $\eta>1$, an $\eta>1$, and an $\varepsilon^{*} \in\left(0, \varepsilon_{1}^{*}\right]$ such that, within $M_{V}\left(\rho^{*}\right)$,

$$
\begin{aligned}
\Delta V(\tilde{x}(k), \tilde{e}(k)) \leq- & \sqrt{\varepsilon} V(\tilde{x}(k), \tilde{e}(k)), \\
& \text { if } V(\tilde{x}(k+s), \tilde{e}(k+s))<\eta V(\tilde{x}(k), \tilde{e}(k)), \forall s \in[-r, 0] .
\end{aligned}
$$

It then follows from Lemma 4 that the closed-loop system (80) is asymptotically stable at the origin with $M_{V}\left(\rho^{*}\right) \supset \Psi$ contained in the domain of attraction.

The proof of Item b) follows the same procedure as in the proof of Item a), but uses the Lyapunov analysis in the proof of Theorems 2 and 4 instead of the analysis in the proof of Theorems 1 and 3. For the validity of these Lyapunov analysis, we establish (78) and (81) without the factor $A^{r}$, which can be easily done by using the fact that $\left|F(\varepsilon) Q(\varepsilon) S^{-1}(\varepsilon)\right| \leq \varepsilon \sum_{i=1}^{l} \alpha_{i}$.

6. Examples. Example 1. Consider the system (1) with

$$
A=\left[\begin{array}{rrrr}
0 & 1 & 0 & 0 \\
0 & 0 & 1 & 0 \\
0 & 0 & 0 & 1 \\
-1 & 2 \sqrt{2} & -4 & 2 \sqrt{2}
\end{array}\right], \quad B=\left[\begin{array}{l}
0 \\
0 \\
0 \\
1
\end{array}\right], \quad C=\left[\begin{array}{llll}
1 & 0 & 0 & 0
\end{array}\right]
$$

The open loop system has two pair of repeated poles on the unit circle at $z= \pm \sqrt{2} / 2 \pm$ $j \sqrt{2} / 2$. Following the proposed design method, we choose

$$
F(\varepsilon)=\left[\begin{array}{llll}
-\varepsilon^{4}+4 \varepsilon^{3}-6 \varepsilon^{2}+4 \varepsilon & -2 \sqrt{2} \varepsilon^{3}+6 \sqrt{2} \varepsilon^{2}-6 \sqrt{2} \varepsilon & -4 \varepsilon^{2}+8 \varepsilon & -2 \sqrt{2} \varepsilon
\end{array}\right] .
$$

Then, the family of linear state feedback laws (25) is given by

$$
u(k)=F(\varepsilon) A^{r} x(k) .
$$


To design an output feedback law, we choose $L=\left[\begin{array}{llll}-2 \sqrt{2} & -4 & -2 \sqrt{2} & 3 / 4\end{array}\right]^{\mathrm{T}}$, which places the eigenvalues of $A+L C$ at $z= \pm 1 / 2 \pm j / 2$, and obtain the family of linear output feedback laws (60) as follows,

(85)

$$
\left\{\begin{aligned}
\hat{x}(k+1) & =\left[\begin{array}{rrrr}
-2 \sqrt{2} & 1 & 0 & 0 \\
-4 & 0 & 1 & 0 \\
-2 \sqrt{2} & 0 & 0 & 1 \\
-1 / 4 & 0 & -2 & 2 \sqrt{2}
\end{array}\right] \hat{x}(t)+\left[\begin{array}{l}
0 \\
0 \\
0 \\
1
\end{array}\right] A^{r} \hat{x}(k-r)-\left[\begin{array}{r}
-2 \sqrt{2} \\
-4 \\
-2 \sqrt{2} \\
3 / 4
\end{array}\right] y(k), \\
u(k) & =F(\varepsilon) A^{r} \hat{x}(k) .
\end{aligned}\right.
$$

Some simulation results of the resulting closed-loop systems are shown in Figs. 2 and 3 .
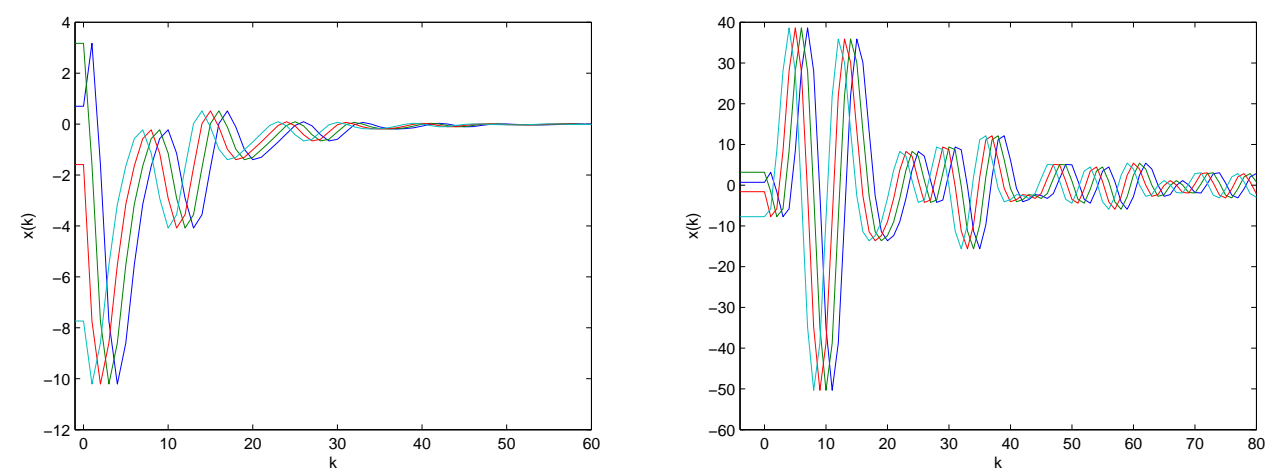

Fig. 2. Example 1. State responses under state feedback law: $r=1$ and $\varepsilon=0.2$ (left plot); $r=4$ and $\varepsilon=0.1$ (right plot).
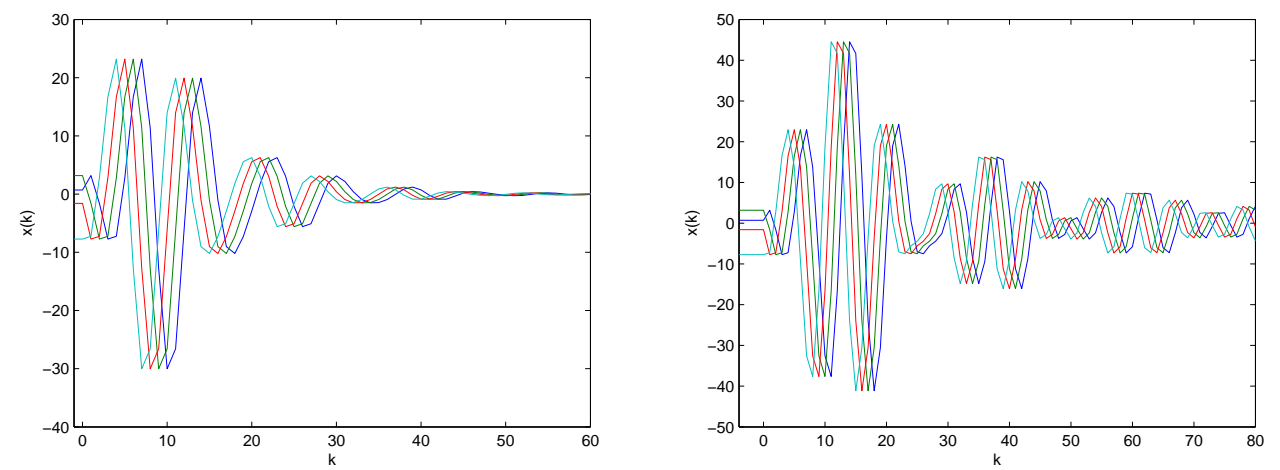

FIG. 3. Example 1. State responses under output feedback law: $r=1$ and $\varepsilon=0.2$ (left plot); $r=4$ and $\varepsilon=0.1$ (right plot).

To verify that these two families of linear feedback laws semi-globally stabilize the given system subject to actuator saturation, we simulate closed-loop systems under 
a given non-zero initial condition for various values of $\varepsilon$. Simulation results (Figs. 4 and 5) indicate that the magnitude of the control input indeed decreases as the value of $\varepsilon$ decreases.
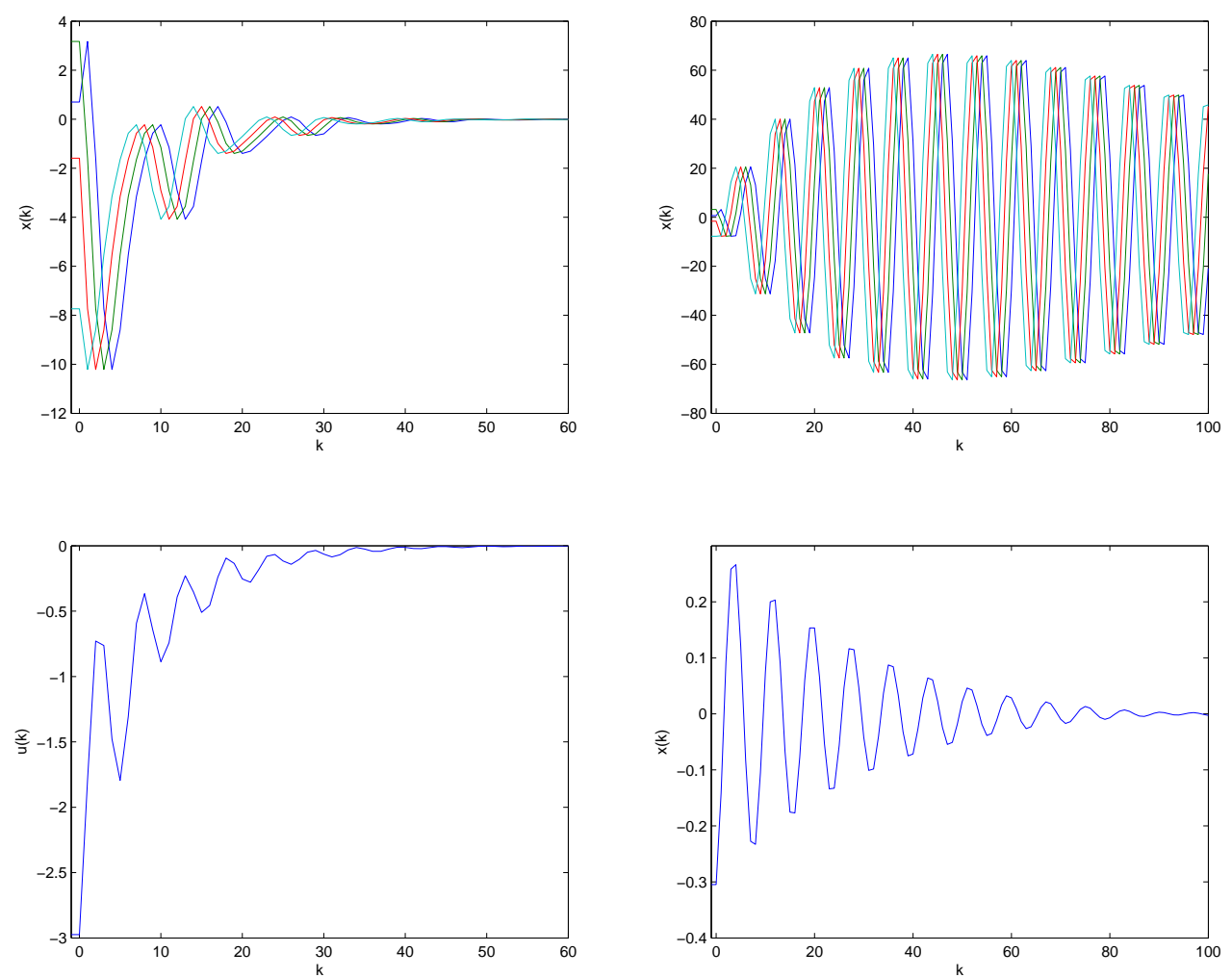

FIG. 4. Example 1. State responses and control input under state feedback law: $r=1$ and $\varepsilon=0.2$ (left plots); $r=1$ and $\varepsilon=0.02$ (right plots).

Example 2. Consider the system (1) with

$$
A=\left[\begin{array}{lllll}
1 & 1 & 0 & 0 & 0 \\
0 & 1 & 1 & 0 & 0 \\
0 & 0 & 1 & 0 & 0 \\
0 & 0 & 0 & 1 & 1 \\
0 & 0 & 0 & 0 & 1
\end{array}\right], \quad B=\left[\begin{array}{rr}
0 & 2 \\
0 & -1 \\
1 & 2 \\
0 & 0 \\
0 & 1
\end{array}\right], \quad C=\left[\begin{array}{lllll}
1 & 0 & 0 & 0 & 0 \\
0 & 0 & 0 & 1 & 0
\end{array}\right]
$$

This system contains two subsystems, each with poles at $z=1$. The two subsystems are coupled through inputs. Then, the family of linear state feedback laws (26) is given by

$$
u(k)=\left[\begin{array}{rrrrr}
-\varepsilon^{3} & -3 \varepsilon^{2} & -3 \varepsilon & 0 & 0 \\
0 & 0 & 0 & -\varepsilon^{2} & -2 \varepsilon
\end{array}\right] x(k) .
$$



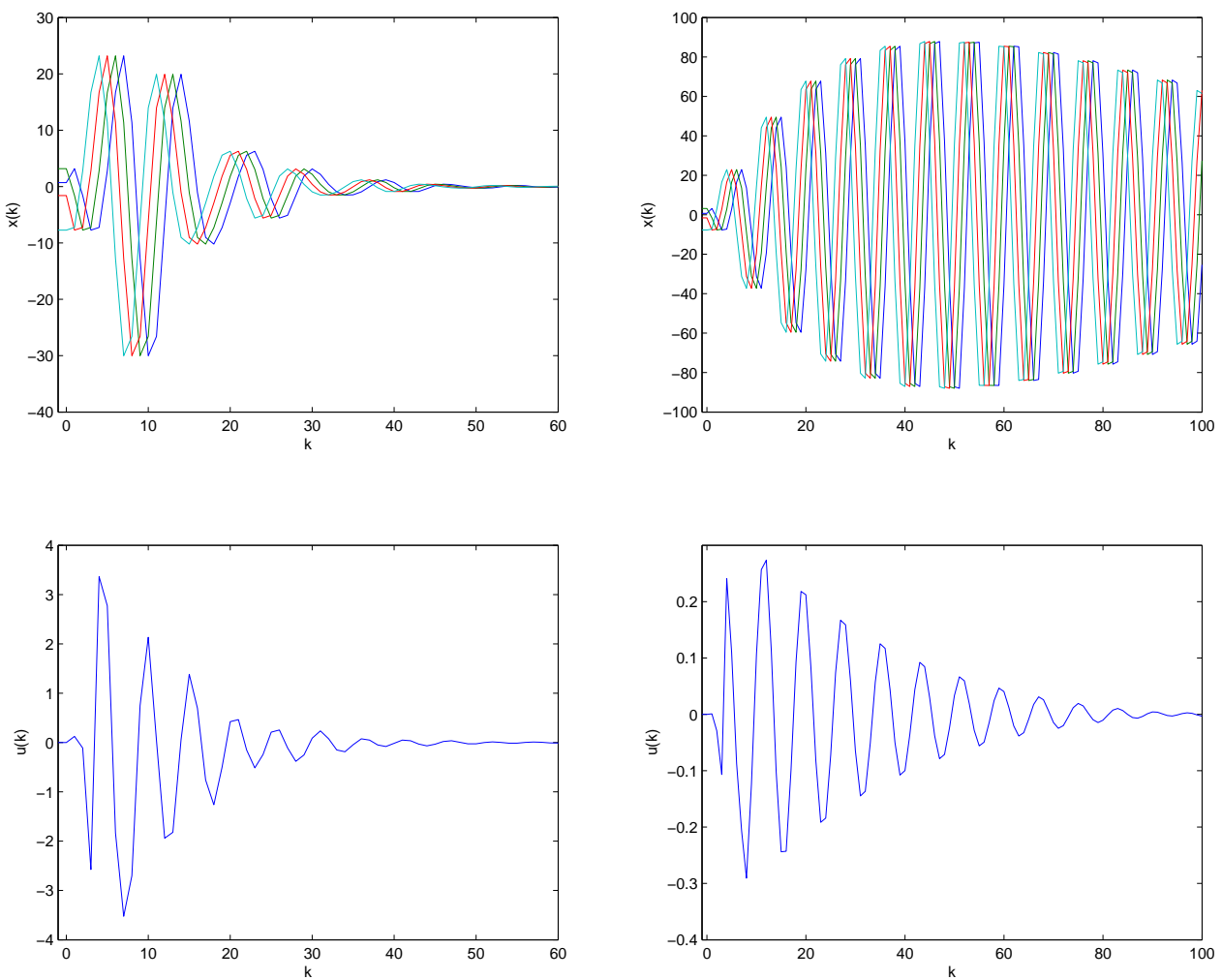

FIG. 5. Example 1. State responses and control input under output feedback law: $r=1$ and $\varepsilon=0.2$ (left plots); $r=1$ and $\varepsilon=0.02$ (right plots).

To design an output feedback law, we choose

$$
L=\left[\begin{array}{rr}
-3 & 0 \\
-3 & 0 \\
-1 & 0 \\
0 & -1 \\
0 & -1 / 4
\end{array}\right]
$$

which places the eigenvalues of $(A+L C)$ at $\{0,0,0,1 / 2,1 / 2\}$. The output feedback 
law (61) can then be computed as

$(88)$

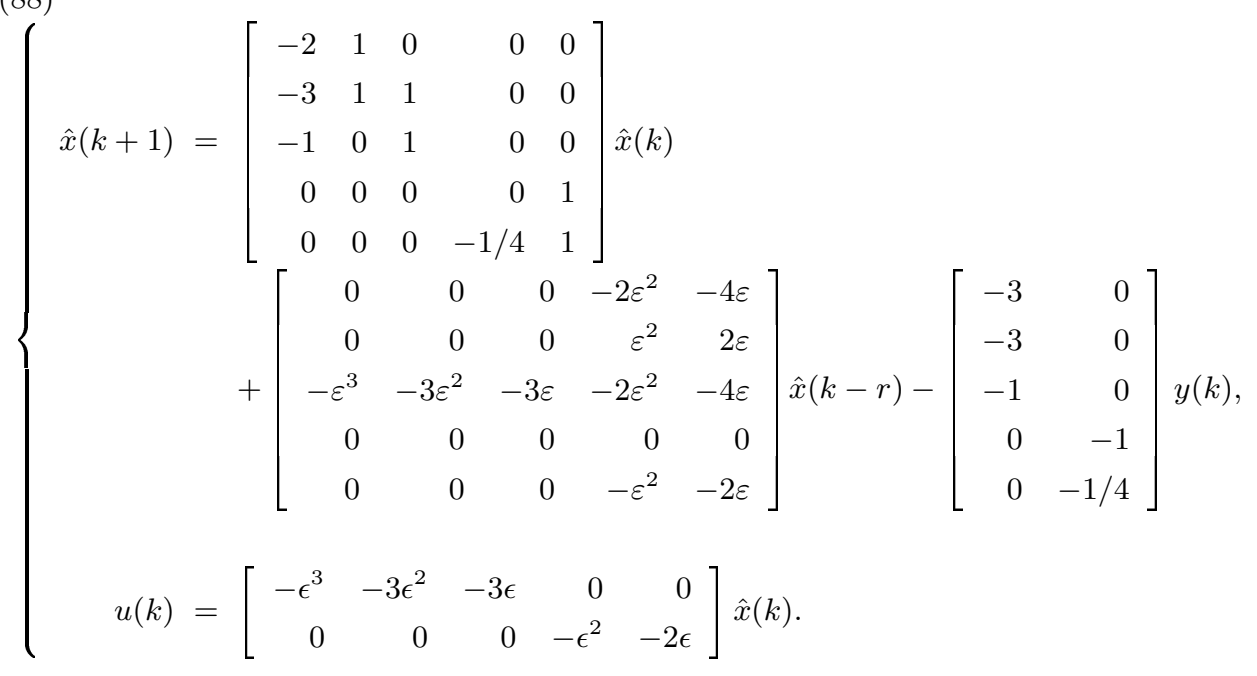

Shown in Figs. 6 and 7 are some simulation results of the resulting closed-loop systems.

To verify that these two families of linear feedback laws semi-globally stabilize the given system subject to actuator saturation, we simulate closed-loop systems under a given non-zero initial condition for various values of $\varepsilon$. Simulation results (Figs. 8 and 9) indicate that the magnitude of the control input indeed decreases as the value of $\varepsilon$ decreases.
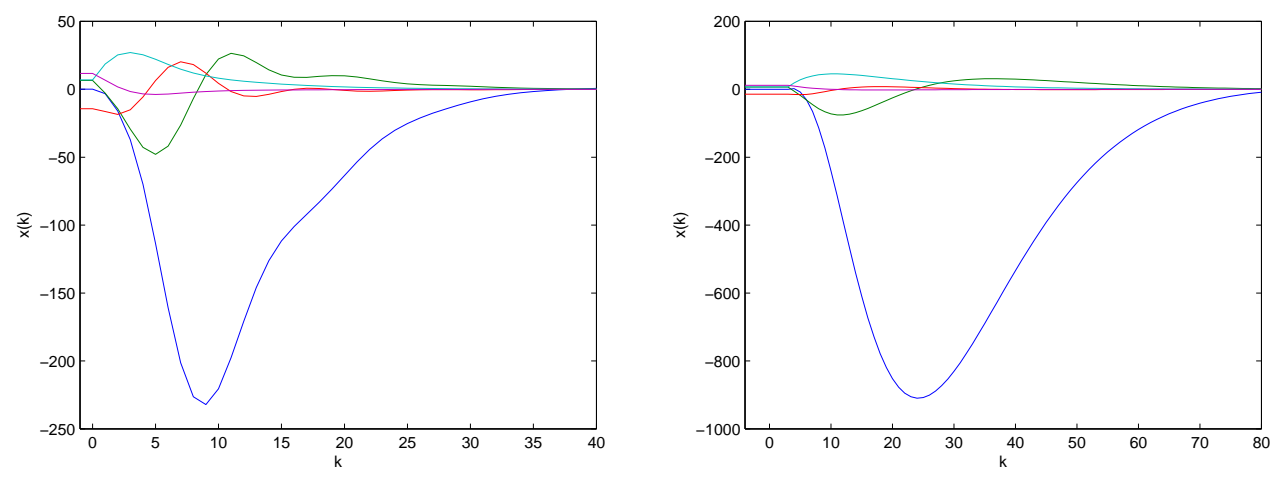

FIG. 6. Example 2. State responses under state feedback law: $r=1$ and $\varepsilon=0.2$ (left plot); $r=4$ and $\varepsilon=0.1$ (right plot).

7. Conclusions. In this paper, we have examined the problem of stabilizing a discrete-time linear system with a delayed linear feedback. We established that a 

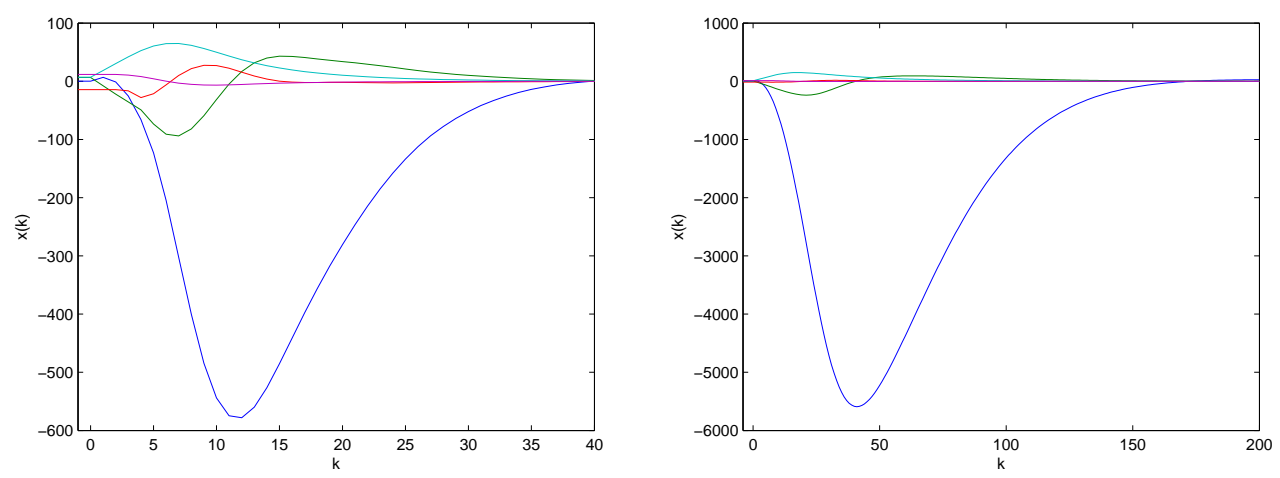

FIG. 7. Example 2. State responses under output feedback law: $r=1$ and $\varepsilon=0.2$ (left plot); $r=4$ and $\varepsilon=0.05$ (right plot).

discrete-time linear system with an arbitrarily large delay in the input can be asymptotically stabilized by either linear state or output feedback as long as the open loop system is not exponentially unstable (i.e., all the open loop poles are on or inside the unit circle). It is further shown that such systems, when subject to actuator saturation, are semi-globally asymptotically stabilizable by linear state or output feedback. Simple examples are constructed to show that these results are not conservative.

\section{REFERENCES}

[1] H.I. Ansoff, Stability for linear oscillating systems with constant time lag, J. Appl. Mech., 16(1949), pp. 158-164.

[2] H.I. Ansoff And J.A. Krumhansl, A general stability criterion for linear oscillating systems with constant time lag, Quart. Appl. Math., 6(1948), pp. 337-341.

[3] Y.-Y. CAO, Z. Lin, AND T. Hu, Stability analysis of linear time-delay systems subject to input saturation, IEEE Trans. Circuits and Systems, 49(2002), pp. 233-240.

[4] B.-S. Chen, S.-S. WAng, AND H.-C. Lu, Stabilization of time-delay system containing saturating actuators, Int. J. Contr., 47(1988), pp. 867-881.

[5] J. Chen and A. Latchman, Frequency sweeping tests for stability independent of delay, IEEE Trans. Auto. Contr., 40:9(1995), pp. 1640-1645.

[6] J.-H. Chou, I.-R. Horng, And B.-S. Chen, Dynamical feedback compensator for uncertain time-delay systems containing saturating actuators, Int. J. Contr., 49(1989), pp. 961-968.

[7] S. Elaydi And S. Zhang, Stability and periodicity of difference equations with finite delay, Funkcialaj Ekvacioj, 37(1994), pp. 401-413.

[8] H. FAng AND Z. Lin, A further result on global stabilization of oscillators with bounded delayed input, IEEE Trans. Auto. Contr., 51:1(2006), pp. 121-128.

[9] K. Gu, V. L. Kharitonov, and J. Chen, Stability of Time-Delay Systems, Birkhäuser, Boston, 2003.

[10] Q. L. HAN AND B. Ni, Delay-dependent robust stabilization for uncertain constrained systems with pointwise and distributed time-varying delays, Proc. 38th IEEE Conf. Decision and Control, pp. 215-220, 1999. 

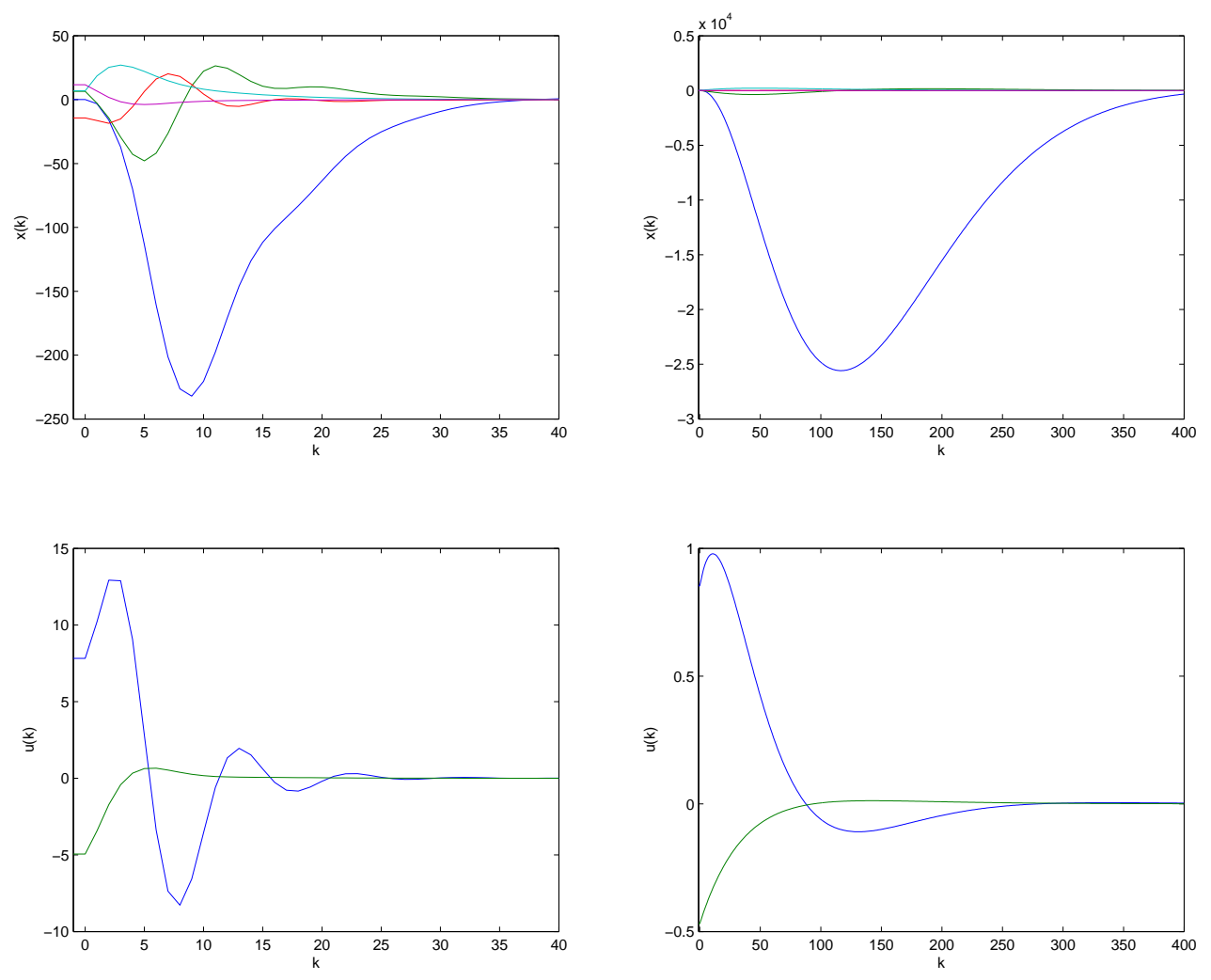

FIG. 8. Example 2. State responses and control input under state feedback law: $r=1$ and $\varepsilon=0.2$ (left plots); $r=1$ and $\varepsilon=0.02$ (right plots).

[11] Z. Lin, B. M. Chen, And X. Liu, Linear Systems Toolkit, Technical Report, Department of Electrical and Computer Engineering, University of Virginia, 2004.

[12] Z. LIN AND H. FANG, On asymptotic stabilizability of linear systems with delayed input, IEEE Transactions on Automatic Control, 52:6(2007), pp. 998-1013.

[13] F. Mazenc, S. Mondie, And S-I Niculescu, Global stabilization of oscillators with bounded delayed input, Systems and Control Letters, 53(2004), pp. 415-422.

[14] F. Mazenc, S. Mondie, And S-I Niculescu, Global asymptotic stabilization for chains of integrators with a delay in the input, IEEE Trans. Auto. Contr., 48(2003), pp. 57-63.

[15] S. Mondié, R. Lozano, And F. Mazenc, Semiglobal stabilization of continuous systems with bounded delayed input, Proc. the 15th IFAC World Congress, Barcelona, Spain, 2002.

[16] S.-I. Niculescu, Delay Effects on Stability: A Robust Control Approach, Springer, Berlin, 2001.

[17] Z. Lin, Low Gain Feedback, Springer, London, 1998.

[18] N. Minorsky, Self-excited oscillations in dynamical systems possessing retarded actions, J. Appl. Mech., 9(1942), pp. A65-A71.

[19] S. Oucheriah, Global stabilization of a class of linear continuous time-delay systems containing saturating controls, IEEE Trans. Circuits Syst. I, 43(1996), pp. 1012-1015.

[20] S. Tarbouriech and J.M. Gomes da Silva JR., Synthesis of controllers for continuous-time delay systems with saturating controls via LMI's, IEEE Trans. Auto. Contr., 45(2000), 

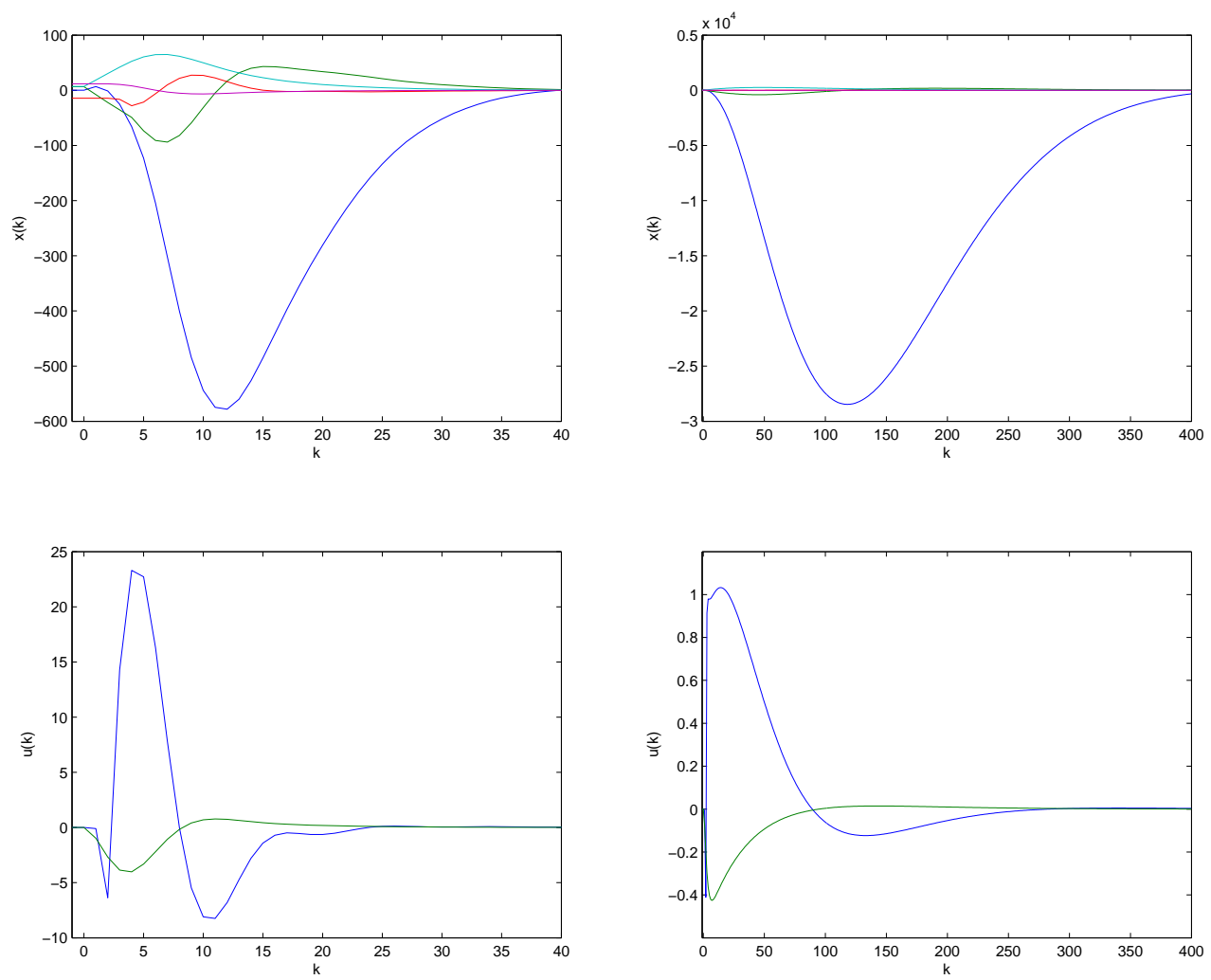

Fig. 9. Example 2. State responses and control input under output feedback law: $r=1$ and $\varepsilon=0.2$ (left plots); $r=1$ and $\varepsilon=0.02$ (right plots).

pp. 105-111.

[21] W. M. Wonham, Linear Multivariable Control: A Geometric Approach, Springer-Verlag, New York, 1979.

[22] M. Wu, Y. He, AND J.-H. ShE, New delay-dependent stability criteria and stabilizing method for neutral systems, IEEE Trans. Auto. Contr., 49(2004), pp. 2266-2271.

[23] L. Xie, E. Fridman and U. Shaked, Robust control of distributed delay systems with application to combustion control, IEEE Trans. Auto. Contr., 46:12(2001), pp. 1930-1935.

[24] S. XIE AND L. XIE, Stabilization of a class of uncertain large-scale stochastic systems with time delays, Automatica, 36(2000), pp. 161-167.

[25] K. Yakoubi And Y. Chitour, Linear systems subject to input saturation and time delay: stabilization and $L_{p}$-stability, Proceedings of the 2nd Symposium on Structure, System and Control, Oaxaca, Mexico, 2004.

[26] H. Zhang, D. Zhang, And L. XIE, An innovation approach to $H_{\infty}$ prediction with application to systems with delayed measurements, Automatica, 40:7(2004), pp. 1253-1261.

[27] S. Zhang And M.-P. Chen, A new Razumikhin Theorem for delay difference equations, Computers Math. Applic., 36:10-12(1998), pp. 405-412. 